\title{
COMPANIONS TO APOGEE STARS. I. A MILKY WAY-SPANNING CATALOG OF STELLAR AND SUBSTELLAR COMPANION CANDIDATES AND THEIR DIVERSE HOSTS
}

\author{
Nicholas W. Troup ${ }^{1}$, David L. Nidever ${ }^{2,3,4}$, Nathan De Lee ${ }^{5,6}$, Joleen Carlberg $^{7}$, STteven R. Majewski $^{1}$,

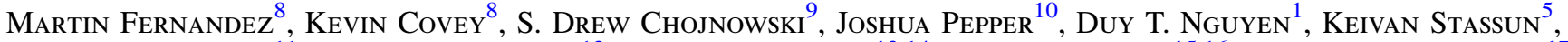 \\ Duy Cuong Nguyen ${ }^{11}$, John P. Wisniewski ${ }^{12}$, Scott W. Fleming ${ }^{13,14}$, Dmitry Bizyaev ${ }^{15,16}$, Peter M. Frinchaboy ${ }^{17}$, \\ D. A. García-Hernández ${ }^{18,19}$, Jian GE ${ }^{20}$, Fred Hearty ${ }^{21,22}$, Szabolcs Meszaros ${ }^{23}$, Kaike Pan ${ }^{15}$, \\ Carlos Allende Prieto ${ }^{18,19}$, Donald P. Schneider ${ }^{21,22}$, Matthew D. Shetrone ${ }^{24}$, Michael F. Skrutskie ${ }^{1}$, \\ JOHN Wilson ${ }^{1}$, AND Olga Zamora ${ }^{18,19}$ \\ ${ }^{1}$ Department of Astronomy, University of Virginia, Charlottesville, VA 22904-4325, USA; nwt2de@virginia.edu \\ ${ }^{2}$ University of Michigan, 1085 S University Ave, Ann Arbor, MI 48109, USA \\ ${ }^{3}$ Large Synoptic Survey Telescope, 950 North Cherry Ave, Tuscon, AZ 85719, USA \\ ${ }^{4}$ Steward Observatory 933 North Cherry Ave, Tuscon, AZ 85719, USA \\ ${ }^{5}$ Department of Physics, Geology, and Engineering Tech, Northern Kentucky University, Highland Heights, KY 41099, USA \\ ${ }^{6}$ Department of Physics and Astronomy, Vanderbilt University, Nashville, TN, USA \\ ${ }^{7}$ NASA Goddard Spaceflight Center, Greenbelt, MD, USA \\ ${ }^{8}$ Western Washington University, Bellingham, WA 98225 , USA \\ ${ }^{9}$ New Mexico State University, Las Cruces, NM, USA \\ ${ }^{10}$ Lehigh University, Bethlehem, PA, USA \\ ${ }^{11}$ University of Toronto, Toronto, Ontario, Canada \\ ${ }^{12}$ University of Oklahoma, Norman, OK, USA \\ ${ }^{13}$ Space Telescope Science Institute, Baltimore, MD, USA \\ ${ }^{14}$ Computer Sciences Corporation, Baltimore, MD, USA \\ 15 Apache Point Observatory and New Mexico State University, P.O. Box 59, Sunspot, NM, 88349-0059, USA \\ ${ }^{16}$ Sternberg Astronomical Institute, Moscow State University, Moscow, Russia \\ ${ }^{17}$ Department of Physics \& Astronomy, Texas Christian University, TCU Box 298840, Fort Worth, TX 76129, USA; p.frinchaboy@tcu.edu \\ ${ }^{18}$ Instituto de Astrofísica de Canarias, Via Láctea s/n, E-38205 La Laguna, Tenerife, Spain \\ ${ }^{19}$ Departamento de Astrofísica, Universidad de La Laguna, E-38206 La Laguna, Tenerife, Spain \\ ${ }^{20}$ Department of Astronomy, University of Florida, Gainesville, FL 32611, USA \\ ${ }^{21}$ Department of Astronomy \& Astrophysics, The Pennsylvania State University, University Park, PA 16802, USA \\ ${ }^{22}$ Center for Exoplanets and Habitable Worlds, The Pennsylvania State University, University Park, PA 16802, USA \\ ${ }^{23}$ ELTE Gothard Astrophysical Observatory, H-9704 Szombathely, Szent Imre Herceg st. 112, Hungary \\ ${ }^{24}$ University of Texas, Austin, TX, USA \\ Received 2015 November 5; accepted 2015 December 28; published 2016 February 29
}

\begin{abstract}
In its three years of operation, the Sloan Digital Sky Survey Apache Point Observatory Galactic Evolution Experiment (APOGEE-1) observed $>14,000$ stars with enough epochs over a sufficient temporal baseline for the fitting of Keplerian orbits. We present the custom orbit-fitting pipeline used to create this catalog, which includes novel quality metrics that account for the phase and velocity coverage of a fitted Keplerian orbit. With a typical radial velocity precision of $\sim 100-200 \mathrm{~m} \mathrm{~s}^{-1}$, APOGEE can probe systems with small separation companions down to a few Jupiter masses. Here we present initial results from a catalog of 382 of the most compelling stellar and substellar companion candidates detected by APOGEE, which orbit a variety of host stars in diverse Galactic environments. Of these, 376 have no previously known small separation companion. The distribution of companion candidates in this catalog shows evidence for an extremely truncated brown dwarf (BD) desert with a paucity of BD companions only for systems with $a<0.1-0.2 \mathrm{AU}$, with no indication of a desert at larger orbital separation. We propose a few potential explanations of this result, some which invoke this catalog's many small separation companion candidates found orbiting evolved stars. Furthermore, 16 BD and planet candidates have been identified around metal-poor $([\mathrm{Fe} / \mathrm{H}]<-0.5)$ stars in this catalog, which may challenge the core accretion model for companions $>10 M_{\mathrm{Jup}}$. Finally, we find all types of companions are ubiquitous throughout the Galactic disk with candidate planetary-mass and BD companions to distances of $\sim 6$ and $\sim 16 \mathrm{kpc}$, respectively.
\end{abstract}

Key words: binaries: close - binaries: spectroscopic - brown dwarfs - Galaxy: stellar content - planetary systems

Supporting material: FITS file

\section{INTRODUCTION}

Over the past few decades, it has been established that solitary Milky Way stars are the exception rather than the rule. Previous studies of stellar multiplicity have shown that more than half of stellar systems contain two or more bound stars, and that stars in these systems span a wide range of separations and mass ratios (e.g., Raghavan et al. 2010; Duchêne \& Kraus 2013). With the advent of the enormous database of confirmed and candidate systems generated by the large-scale planet-hunting mission Kepler (Borucki et al. 2010), planetary companions are also thought to be quite commonplace, including an unexpected class of short-period Jupiter-mass planets, the first discovered by Mayor \& Queloz (1995). These "hot Jupiters," have been explained by inward orbital migration during their formation (Masset \& Papaloizou 2003). Interestingly, while both exoplanets and stellar-mass companions have 
been found in extremely short-period orbits, there has been a paucity of brown dwarf $(\mathrm{BD})^{25}$ companions orbiting Sun-like stars, a phenomenon known as the "brown dwarf desert" (Marcy \& Butler 2000). However, more recent work has shown that this desert might be limited in extent, with no desert for wide ( $a<1000$ AU) companions (Gizis et al. 2001), and may not be as "dry" as initially thought when considering stars more massive than the Sun (Guillot et al. 2014).

Traditionally, solar-like dwarf stars have been the primary targets for exoplanet searches and stellar multiplicity studies. However, recently some work has been done with evolved stars (e.g., Reffert et al. 2006; Johnson et al. 2007; Lovis \& Mayor 2007; Wittenmyer et al. 2011; Zieliski et al. 2012). Currently, there are only approximately 50 known planet-hosting giant stars, compared to the $>1000$ known dwarf-star planet hosts (Jones et al. 2014a), but even this small sample of giant star hosts has produced some interesting results. As a star like the Sun expands into a red giant, its atmosphere will engulf the innermost planets (e.g., Villaver \& Livio 2009; Villaver et al. 2014). Stronger tidal dissipation from the expanding star may also lead to more distant companions also being consumed. Possible observational signatures of planetary engulfment have been identified in the chemical abundances and peculiarly high rotational velocities seen in some giant stars (e.g., Massarotti et al. 2008; Adamów et al. 2012; Carlberg et al. 2012). However, Silvotti et al. (2014) have found hot Jupiters orbiting subdwarf B stars, which suggests that some Jovian planets may survive within the extended envelope of their host star during its red giant phase.

It is becoming clear that the properties of the host star plays an important role in the types of companions that can form with it. It has been established that metal-rich host stars are more likely to host Jovian planets than their metal-poor counterparts (Fischer \& Valenti 2005). This relation is believed to be a consequence of the core accretion model of planet formation, which requires a potential Jovian planet to acquire $\sim 5-10 M_{\oplus}$ worth of solid material before the central star expels the hydrogen and helium gas from the protoplanetary disk (Matsuo et al. 2007). Similar trends relating individual elemental abundances to planet occurrence rate have also been found (e.g., Bodaghee et al. 2003; Robinson et al. 2006; Adibekyan et al. 2012). Stellar binaries are formed via a separate mechanism, and it is disputed whether or not metallicity plays a role in binary fraction (Abt 2008). Binarity has generally been found to be higher in lower metallicity populations (e.g., Carney et al. 2003). However, a higher fraction of stellar binaries has been found among metal-rich F-type dwarfs in the field compared to their metal-poor counterparts (Hettinger et al. 2015). It is not clear whether BD formation follows star or planet formation trends more closely. Planet occurrence rate has also been shown to depend on the mass of the host star, with higher-mass hosts being less likely to host a planet than lower-mass hosts (e.g., Reffert et al. 2015).

Most exoplanet and multiplicity surveys have also focused on targeting stars within the solar neighborhood because of the aforementioned concentration on solar-like dwarf stars, and the greater difficulty in measuring transit signals and radial velocities (RVs) for these types of stars at great distances. Because of these limitations, there is a limited understanding of

\footnotetext{
${ }^{25}$ For this paper we define a BD companion as a companion with a mass between the Deuterium-burning $\left(0.013 M_{\odot}\right)$ and Hydrogen-burning $\left(0.080 M_{\odot}\right)$ limits.
}

the Galactic distribution of companions. Microlensing surveys such as The Optical Gravitational Lensing Experiment (OGLE; Udalski 2003) have discovered potential planetary-mass candidates in the Galactic Bulge (Shvartzvald et al. 2014), but few other planets have been found farther than $\sim 1 \mathrm{kpc}$ from the Sun. Furthermore, the vast majority of planets have been identified among Galactic field stars, while only a few planets have been discovered in open clusters (e.g., Lovis \& Mayor 2007; Brucalassi et al. 2014).

\subsection{The Role of APOGEE}

Many of the aforementioned discoveries came through small and large-scale stellar transit monitoring, the use of singleobject spectroscopy, or the combination thereof. A logical step forward in this field is the use of large-scale multi-object spectroscopy to complement current and future large photometric surveys such as those by Kepler (Borucki et al. 2010) and TESS (Ricker et al. 2014). The Sloan Digital Sky Survey III (SDSS-III; Eisenstein et al. 2011) Multi-object APO Radial Velocity Exoplanet Large-area Survey (MARVELS; Ge et al. 2008) used this approach to observe $\sim 10,000$ stars and discovered several BD and low-mass stellar companions (Lee et al. 2011; Fleming et al. 2012; Wisniewski et al. 2012; De Lee et al. 2013; Jiang et al. 2013; Ma et al. 2013; Mack et al. 2013; Wright et al. 2013).

The SDSS-III Apache Point Observatory Galactic Evolution Experiment (APOGEE Majewski et al. 2015) is a large-scale, systematic, high-resolution $(R=22,500), H$-band $(1.51 \mu \mathrm{m}<\lambda<1.69 \mu \mathrm{m})$, spectroscopic survey of the chemical and kinematical distribution of Milky Way stars. APOGEE acquired high signal-to-noise ratio $(\mathrm{S} / \mathrm{N})(>100)$ spectra of over 146,000 stars distributed across the Galactic bulge, disk, and halo. To achieve this $\mathrm{S} / \mathrm{N}$, many of the stars had to be observed for long net integration times-up to $24 \mathrm{hr}$. To accomplish this goal, and to gain sensitivity to temporal variations in $\mathrm{RV}$ indicative of stellar companions, the APOGEE survey observed most stars over multiple epochs. In three years of operations, APOGEE observed over 14,000 stars enough times $(\geqslant 8)$ and over a sufficient temporal baseline to collect spectra yielding high quality RV measurements suitable to not only reliably detect RV variability, but also to construct reliable Keplerian orbital fits to search for companions of a wide range of masses. With a typical RV precision of $\sim 100-200 \mathrm{~m} \mathrm{~s}^{-1}$, APOGEE can detect RV oscillations typical of those expected from relatively short-period companions down to a few Jupiter-masses $\left(10^{-3} M_{\odot}\right)$. And because of APOGEE's design as a systematic probe of Galactic structure, this sample probes stellar populations not traditionally sought in exoplanet and stellar multiplicity studies in regions of the Milky Way well beyond the solar neighborhood.

\subsection{Paper Overview}

In this paper, we present the first catalog of 382 candidate companions detected by APOGEE. In Section 2, we give a brief description of the nature of the APOGEE observations, with a general description of the APOGEE data reduction in Section 3. Section 3 also introduces the apOrbit pipeline, describing how the RVs and orbital parameters are derived, and introduces novel quality criteria which quantifies and accounts for both the phase and velocity space coverage of the fitted Keplerian model. Section 4 presents APOGEE's first catalog of 
candidate companions to stars observed by APOGEE, and in particular, describes how we select the statistically significant RV variable sample, and the final "gold sample" of candidate companions. In Section 5, we discuss global analysis of this gold sample. Finally, in Section 6 we describe planned future efforts with this and future, expanded catalogs, and we summarize conclusions drawn from the gold sample in Section 7. Verification efforts of the aporbit pipeline are described in Appendix A, and instruction on how to access and use the catalog are presented in Appendix B.

\section{APOGEE RV OBSERVATIONS}

All APOGEE-1 observations were taken using fibers connected to either the Sloan $2.5 \mathrm{~m}$ telescope (Gunn et al. 2006) or the NMSU $1 \mathrm{~m}$ telescope at Apache Point Observatory (APO; Majewski et al. 2015). In normal use on the Sloan $2.5 \mathrm{~m}$ telescope, APOGEE employs a massively multiplexed, fiber-fed spectrograph capable of recording 300 spectra at a time. For full details on the APOGEE instrument see J. Wilson et al. (2016, in preparation).

Of the 146,000 stars observed in APOGEE-1, 14,840 had at least eight visits; these stars were selected for analysis here. APOGEE first light observations were obtained in 2011 May and APOGEE-1 observations concluded at the end of SDSS-III in 2014 July, providing a maximum temporal baseline of slightly more than three years ( 1000 days). Figure 1 shows the distribution of temporal baselines for stars submitted for Keplerian orbit fitting, as well as the distribution of the number of visits to each of these stars. An APOGEE "visit" is defined as the combined spectrum of a source from a single night's observations, typically $\sim 1 \mathrm{hr}$ of exposure. For main survey targets, the number of visits scheduled for a star depends on its $H$ magnitude, with fainter targets needing more visits to acquire the APOGEE target accumulated $\mathrm{S} / \mathrm{N}$ of 100 per halfresolution element. For stars with at least eight visits, individual visit spectra obtained a median $\mathrm{S} / \mathrm{N}$ of 12.2 . Visits are required to be separated by $\geqslant 3$ days, and must span $\geqslant 30$ days at minimum to gauge the potential binarity of the source. Special targets such as stars used for calibration or ancillary science programs often have additional visits and employ a non-standard cadence. For example, some stars observed during commissioning were re-observed at the end of the survey as a consistency check (see Appendix B.1), so these stars may have visits separated by over two years. For a more detailed description of APOGEE targeting and observing strategy see Zasowski et al. (2013) and Majewski et al. (2015).

\section{DATA REDUCTION AND THE APORBIT PIPELINE}

Because the results of the present work depend critically on an understanding of the RVs and their uncertainties, we first review those aspects of the data reduction process most relevant to the derivation of the RVs. For more information on processing steps that lead to the creation of the individual visit spectra, as well as more information regarding the main APOGEE data reduction pipeline (apogeereduce) see Nidever et al. (2015).

After producing the individual visit spectra, apogeereduce performs initial RV corrections on the visit spectra (described briefly in Section 3.1), and combines them into a single spectrum for each star. The APOGEE Stellar Parameters and Chemical Abundances pipeline (ASPCAP; García Pérez
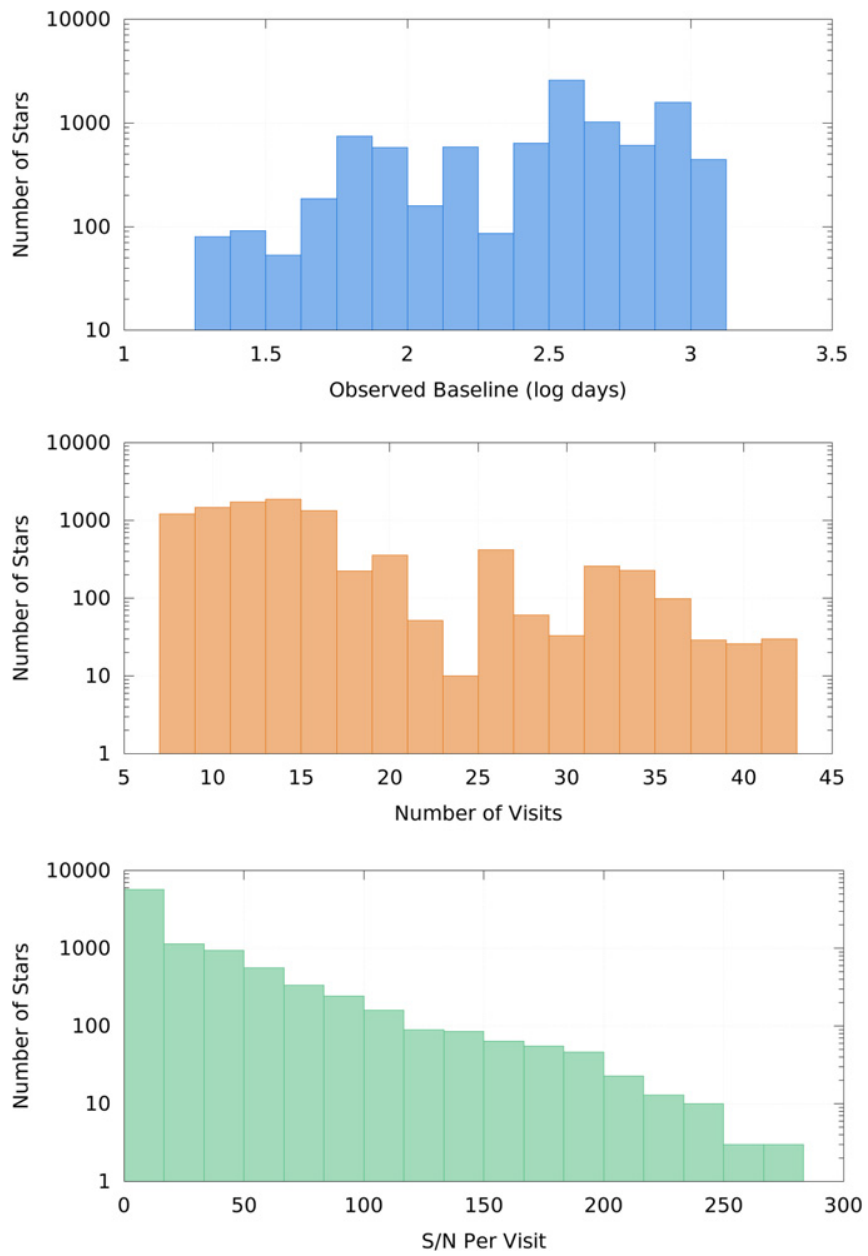

Figure 1. Top Panel: distribution of the observed baseline for the 14,840 stars with at least eight visits. The median baseline for this set of stars is slightly over a year at 384 days. Middle Panel: distribution of the number of visits to the same set of stars, with 13 being the median number of visits. Bottom Panel: distribution of the average $\mathrm{S} / \mathrm{N}$ per visit for the same set of stars, with a median $\mathrm{S} / \mathrm{N}$ per visit of 12.2 .

et al. 2015) then matches this combined spectrum to a library of synthetic spectra (Zamora et al. 2015), constructed by using extensive atomic/molecular linelists (Shetrone et al. 2015), automatically delivering accurate stellar atmospheric parameters $\left(T_{\text {eff }}\right.$ within $\sim 100 \mathrm{~K}, \log g$ and $[\mathrm{Fe} / \mathrm{H}]$ within $\sim 0.1$ dex) and the abundances of up to 15 chemical elements (Fe, C, N, O, $\mathrm{Na}, \mathrm{Mg}, \mathrm{Al}, \mathrm{Si}, \mathrm{S}, \mathrm{K}, \mathrm{Ca}, \mathrm{Ti}, \mathrm{V}, \mathrm{Mn}, \mathrm{Ni})$. Both the model synthetic spectrum and stellar parameters derived for the star are used in the production of the final RVs used in orbit fitting as described in Section 3.1 and to derive the properties for the primary star as described in Section 3.2.

\subsection{Derivation of $R V s$}

The main APOGEE pipeline retains RVs from two methods: (1) the APOGEE reduction pipeline initially selects, through $\chi^{2}$ minimization, an RV template from a coarse grid of synthetic spectra (the "RV mini-grid"). This template is cross-correlated against the spectrum to produce absolute RVs. (2) The pipeline cross-correlates the visit spectra with a combined spectrum of all visits and applies a barycentric correction to acquire heliocentric RVs. These RVs are stored as APOGEE data products. 

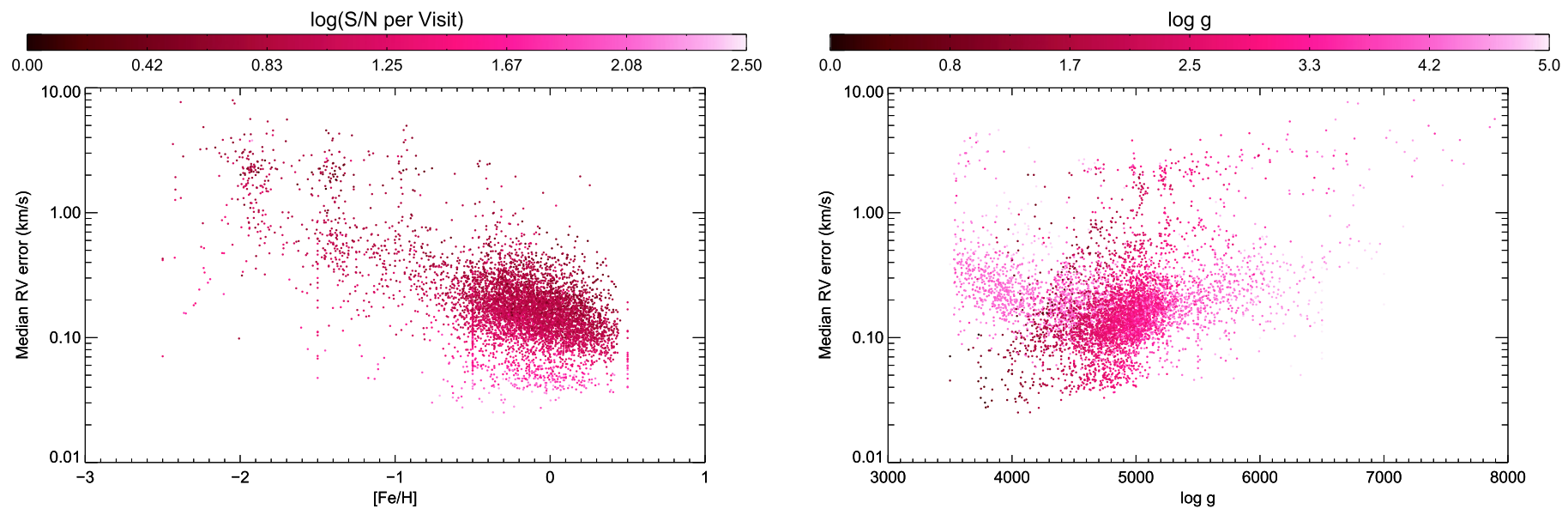

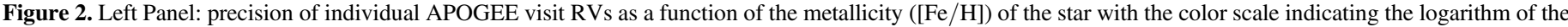

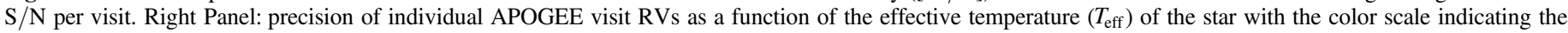
surface gravity $(\log g)$ of the star.

To ensure the highest precision RVs, we preformed the additional step of using the best-fit synthetic spectrum chosen by ASPCAP as the RV template. The grid of synthetic spectra used by ASPCAP is much finer than the RV mini-grid with additional dimensions to account for $[\alpha / \mathrm{M}],[\mathrm{C} / \mathrm{M}]$, and $[\mathrm{N} /$ $\mathrm{M}]$. In addition, the final model spectrum is achieved through cubic Bézier interpolation in the grid of spectra. Therefore, the ASPCAP best-fit template is a significant improvement over the RV mini-grid template and provides a high-quality match to the observed combined spectrum. This approach combines the advantages of using a noiseless synthetic spectrum as a template and using the combined observed spectrum to mitigate the chances of template mismatch. In the cases when mismatch did occur (e.g., due to a poor or failed ASPCAP solution), we deferred to the RVs derived from the combined observed spectrum template. In either case, the RVs we used for orbit fitting were heliocentric RVs.

\subsubsection{Analysis of RV Precision}

To fully understand the types of companions to which we are sensitive, we need a clear understanding of dependencies of the RV precision on stellar parameters. Therefore, we created an empirical model of the RV precision based on the primary derived stellar parameters $\left(T_{\mathrm{eff}}, \log g,[\mathrm{Fe} / \mathrm{H}]\right)$ and the $\mathrm{S} / \mathrm{N}$ for each visit of the star:

$$
\begin{aligned}
\log \sigma_{v}= & 1.56+\left(4.87 \times 10^{-5}\right) T_{\text {eff }}+0.135 \log g \\
& -0.518[\mathrm{Fe} / \mathrm{H}]-\left(5.55 \times 10^{-3}\right) \mathrm{S} / \mathrm{N},
\end{aligned}
$$

where $\mathrm{S} / \mathrm{N}$ is the signal-to-noise ratio of the visit spectrum from which the RV measurement was derived, and $\sigma_{v}$ is the RV measurement error in $\mathrm{ms}^{-1}$. This model was determined by fitting a linear function of each parameter of interest using all APOGEE stars with at least 8 visits, excluding stars used as telluric standards and stars that have unreliable stellar parameters. The left panel of Figure 2 displays two of the stronger effects on RV error: $[\mathrm{Fe} / \mathrm{H}]$ and $\mathrm{S} / \mathrm{N}$ per visit. The effects of $\log g$ and $T_{\text {eff }}$ are illustrated in the right panel. These effects are closely related to the strength and number of absorption lines in the spectra. For a typical solar metallicity $([\mathrm{Fe} / \mathrm{H}]=0)$ giant $\left(T_{\text {eff }}=4000 \mathrm{~K}, \log g=3\right)$ and typical solar metallicity dwarf $\left(T_{\text {eff }}=5000 \mathrm{~K}, \log g=4.5\right)$ stars with $\mathrm{S} / \mathrm{N}=10$, we derive a typical $\mathrm{RV}$ precision of $\sim 130 \mathrm{~m} \mathrm{~s}^{-1}$ and $\sim 230 \mathrm{~m} \mathrm{~s}^{-1}$, respectively per visit. These are the random RV uncertainties reported by the APOGEE pipeline, and are likely to be underestimates of the true uncertainty (see Appendix B.1).

\subsubsection{Selection of Usable RVs and RV Variable Stars}

$\mathrm{RV}$ measurements from observations with $\mathrm{S} / \mathrm{N}<5$, as well visits that produced failure conditions in the RV pipeline, were not included in the final RV curves submitted to the orbit fitter. This reduced the number of stars for which Keplerian orbits could be attempted from 14,840 to 9454 stars.

Likely RV variable stars were selected using the following statistic:

$$
\Sigma_{\mathrm{RV}}=\operatorname{stddev}\left(\frac{\boldsymbol{v}-\tilde{v}}{\boldsymbol{\sigma}_{v}}\right) \geqslant 2.5,
$$

where $v$ and $\sigma_{v}$ are the RV measurements and their uncertainties, and $\tilde{v}$ is the median RV measurement for the star. The criterion was motivated by the false positive analysis presented in Appendix A.1.2. There are also several additional pieces of information that we used to pre-reject stars that would have resulted in poor or erroneous Keplerian orbit fits. Therefore we also removed stars with the following criteria:

1. The system's primary must be characterized with reliable stellar parameters $\left(T_{\text {eff }}, \log g,[\mathrm{Fe} / \mathrm{H}]\right)$, so the ASPCAP STAR_BAD flag must not be set for the star. Derivations of the RVs and the physical parameters of the system both rely on reasonable estimates of the stellar parameters of the host star.

2. The star cannot have been used as a telluric standard. These stars are selected for APOGEE observation for their nearly featureless spectra, so it is likely that RVs derived for these stars are unreliable and would lead to false positive signals.

3. The combined spectrum from which the stellar parameters and RVs were derived cannot be contaminated with spurious signals due to poor combination of the visit 

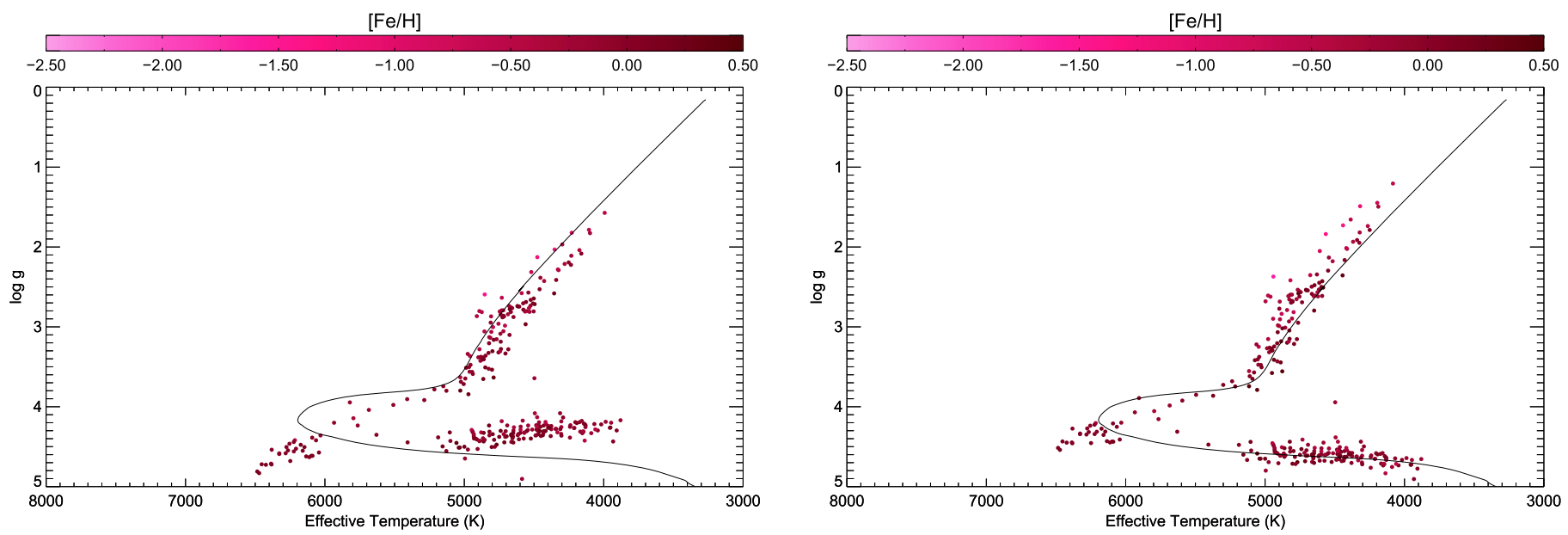

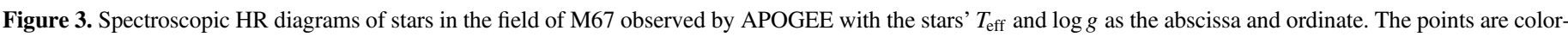

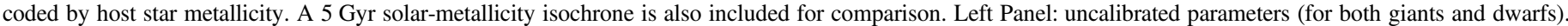

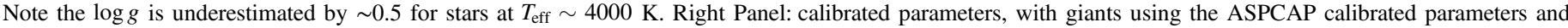
dwarfs adopting the $\log g$ correction from Equation (3).

spectra, so the SUSPECT_RV_COMBINATION flag must not be set for the star. This criterion also catches the double-lined spectroscopic binaries (SB2s) that would have resulted in poor stellar parameters, RVs, or orbital parameters from our current pipelines.

This preselection reduced the number of stars for which Keplerian orbit fits were attempted from 9454 to 907 . This is not to say the stars excluded do not have any sort of RV variation, but the false positive interpretation cannot be ruled out for these stars, so we elected not to include them.

\subsection{Derivation of Primary Stellar Parameters}

To determine masses of potential companions, a reasonable estimate of the primary star's mass is required. The measurement of masses for the primary stars in this sample is based on the spectroscopic stellar parameters $\left(T_{\mathrm{eff}}, \log g,[\mathrm{Fe} / \mathrm{H}]\right)$ derived for each star. Between apogeereduce and ASPCAP, stellar parameters are derived up to three times for each source. The first approach uses the stellar parameters from the RV template selected for determining initial visit-level RVs. These parameters are available for every star, but are also the least precise of the three methods, so they should only be used as a last resort. The next set of stellar parameters made available are from the raw ASPCAP output. Except in the rare cases where ASPCAP fails to converge (which are removed from the final sample), these are available for all stars. Finally, calibrations are applied to the raw ASPCAP results based on comparisons with manual analysis of cluster stars (Mészáros et al. 2013; Holtzman et al. 2015). These parameters are only available for giant stars in a specific temperature range $\left(3500<T_{\text {eff }}<6000\right.$ $\mathrm{K})$, but are the most reliable in absolute terms. To summarize, in order of preference, we adopted: (1) stellar parameters from the calibrated ASPCAP parameters, (2) uncalibrated ASPCAP parameters, (3) parameters used by the much coarser RV mini-grid.

All of the dwarfs in this catalog rely on uncalibrated parameters. Unfortunately this leads to systematically overestimated $\log g$ values for cool dwarfs when compared to Dartmouth isochrones (Figure 3). We apply a simple linear correction to calibrate dwarf $\log g$ values:

$$
(\log g)_{\mathrm{cal}}=\log g-\left(3 \times 10^{-4}\right)\left(T_{\mathrm{eff}}-5500 \mathrm{~K}\right),
$$

where $\log g$ and $T_{\text {eff }}$ are the uncalibrated suface gravity and effective temperature. The results of this calibration can be seen in Figure 3.

\subsubsection{Primary Star Classification}

Before any further stellar properties are estimated, we divide the stars in this sample into 5 classes defined by the following crteria:

1. Pre-main Sequence (PMS): Stars flagged in APOGEE as young stellar cluster members (IC 348 and Orion).

2. Red Clump $(R C)$ : Stars in the APOGEE RC Catalog (Bovy et al. 2014).

3. Red Giant $(R G)$ : Stars not selected as RC or PMS stars with

$$
\begin{aligned}
T_{\text {eff }} & <5500 \mathrm{~K}, \\
\log g & <3.7+0.1[\mathrm{Fe} / \mathrm{H}] .
\end{aligned}
$$

The second relation was derived by mapping the $\log g$ of the base of the giant branch as a function of $[\mathrm{Fe} / \mathrm{H}]$ from Dartmouth isochrones (Chaboyer et al. 2008) for typical ages expected of APOGEE giants.

4. Subgiant $(S G)$ : Stars not selected as RC or PMS stars with

$$
\begin{aligned}
T_{\text {eff }} & >4800 \mathrm{~K}, \\
\log g & \geqslant 3.7+0.1[\mathrm{Fe} / \mathrm{H}], \\
\log g & \leqslant 4-\left(7 \times 10^{-5}\right)\left(T_{\text {eff }}-8000 \mathrm{~K}\right) .
\end{aligned}
$$

The second relation only applies for $T_{\text {eff }}<5500 \mathrm{~K}$. The third relation was determined by the $\log g$ at the highest $T_{\text {eff }}$ of Dartmouth isochrones at a variety of ages and [Fe/ $\mathrm{H}]$, roughly mapping the main-sequence turnoff (MSTO), and fitting a liner function to these points.

5. Dwarf (MS): Any star that does not fit into any of the above categories are classified as MS stars.

These classifications are saved for the catalog, and illustrated in Figure 4. 


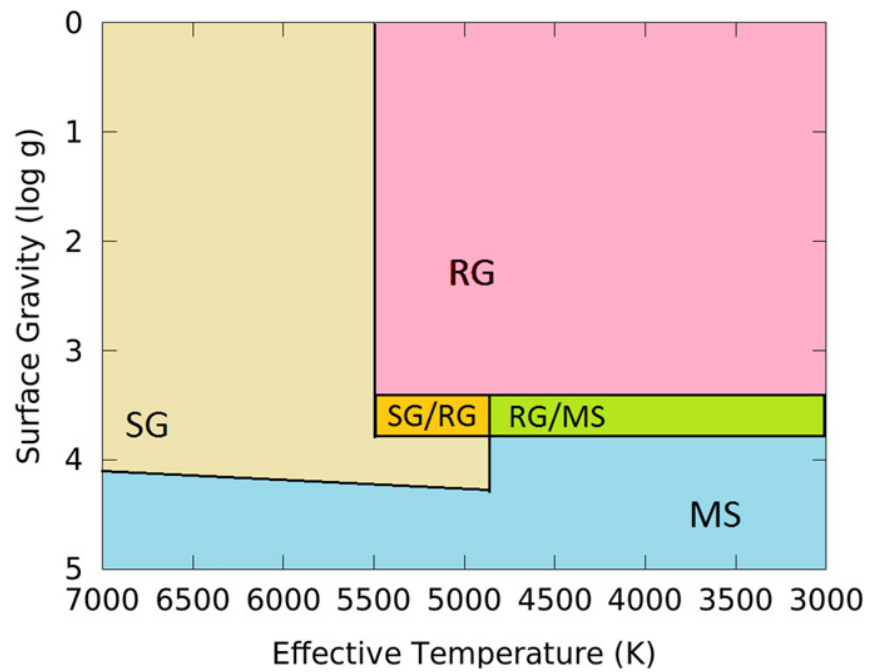

Figure 4. Classification scheme of Red Giant (RG), Subgiant (SG), and mainsequence dwarf stars (MS) in $\log g-T_{\text {eff }}$ space. Red Clump (RC) and pre-main sequence stars (PMS) transcend these boundries as they selected through alternate means. The areas labeled with $\mathrm{SG} / \mathrm{RG}$ or $\mathrm{MS} / \mathrm{RG}$ are regions where the star can be either classification depending on its metallicity. The upper left corner of this plot does not contain any stars in this sample, so the SG classification there is simply in place to cover the phase space.

\subsubsection{Derivation of Bolometric Magnitudes}

In addition to stellar parameters, we need an estimate of the stars' bolometric magnitudes to compare to the bolometric luminosities we calculate and use in the following derivations of the masses and radii of the primary stars. We adopt the extinction coefficient, $A_{K}$ from the APOGEE targeting data (Zasowski et al. 2013). If the APOGEE targeting $A_{K}$ is not populated or is less than zero, then we adopt the WISE all-sky $K$-band extinction. In the rare case $(<1 \%$ of stars run through the apOrbit pipeline) that neither quantity is available, we assume $A_{K}=0$, and flag the star. The extinction-corrected $K_{s}$ magnitude is then $K_{0}=K_{s}-A_{K}$. We derived the bolometric correction to the 2MASS $K_{s}$ band from Dartmouth isochrones:

$$
\begin{aligned}
\mathrm{BC}_{K}= & (2.7+0.15[\mathrm{Fe} / \mathrm{H}]) \\
& -(25+0.5[\mathrm{Fe} / \mathrm{H}]) X^{2-0.1[\mathrm{Fe} / \mathrm{H}]} e^{-X}
\end{aligned}
$$

for PMS, dwarf and SG stars, where $X=\log T_{\text {eff }}-3.5$, and

$$
\mathrm{BC}_{K}=(6.8-0.2[\mathrm{Fe} / \mathrm{H}])\left(3.96-\log T_{\mathrm{eff}}\right)
$$

for RG and RC stars. This correction yields the bolometric magnitude of the star: $m_{\mathrm{bol}}=K_{0}+\mathrm{BC}_{K}$.

\subsubsection{Derivation of Dwarf and Subgiant Primary Mass, Radius, and Distance}

For stars selected as dwarf and subgiant stars, we adopted the Torres et al. (2010) relations to estimate the mass and radius of the primary star:

$$
\begin{aligned}
\log M_{\star}= & a_{1}+a_{2} X+a_{3} X^{2}+a_{4} X^{3} \\
& +a_{5}(\log g)^{2}+a_{6}(\log g)^{3}+a_{7}[\mathrm{Fe} / \mathrm{H}], \\
\log R_{\star}= & b_{1}+b_{2} X+b_{3} X^{2}+b_{4} X^{3} \\
& +b_{5}(\log g)^{2}+b_{6}(\log g)^{3}+b_{7}[\mathrm{Fe} / \mathrm{H}],
\end{aligned}
$$

where $X=\log T_{\text {eff }}-4.1$ and the coefficients, $a_{i}$ and $b_{i}$ are given in Table 4 of Torres et al. (2010). This empirical relationship has a scatter of $6.4 \%$ in mass and $3.2 \%$ in radius, so for dwarfs and subgiants, we adopt $\sigma_{M_{\star}}=0.064 M_{\star}$ as the uncertainty in the mass, and $\sigma_{R_{\star}}=0.032 R_{\star}$. This information allows one to estimate the luminosity, $L_{\star}$, as well as the distance, $d$, to these stars:

$$
\begin{gathered}
L_{\star}=4 \pi R_{\star}^{2} \sigma_{\mathrm{SB}} T_{\mathrm{eff}}^{4} \\
M_{\mathrm{bol}}=4.77-2.5 \log \left(\frac{L_{\star}}{L_{\odot}}\right) \\
d=10^{1+0.2\left(m_{\mathrm{bol}}-M_{\mathrm{bol}}\right)},
\end{gathered}
$$

where $M_{\mathrm{bol}}$ is the star's absolute bolometric magnitude. Uncertainty for these parameters are also derived through normal propagation of uncertainties, which yields a $13.5 \%$ typical distance uncertainty for dwarfs and subgiants. A total of 340 of the 907 stars for which fitting was attempted used this prescription.

Unfortunately, the Torres et al. (2010) relations are not applicable to giant and PMS stars. For example, using the Torres et al. (2010) relations to derive the mass of Arcturus $\left(T_{\text {eff }}=4286 \mathrm{~K}, \log g=1.66,[\mathrm{Fe} / \mathrm{H}]=-0.52\right)$ yields a mass of $3.5 M_{\odot}$ compared to the accepted mass of $1.08 M_{\odot}$ (Ramírez \& Allende Prieto 2011). Therefore, we must resort to alternate methods for estimating the mass of the primary.

\subsubsection{Derivation of Giant and PMS Primary Mass, Radius, and Distance}

Efforts are currently underway to compile all published (or soon-to-be published) distance measurements to APOGEE stars. For stars selected as RG and RC stars, we employ a preliminary version of this distance catalog as the basis for our mass derivation. The most accurate distances for APOGEE stars are those derived from asteroseismic parameters from the APOGEE-Kepler catalog (APOKASC; Pinsonneault et al. 2014). These distances were given first priority because they only have $\sim 2 \%$ random errors (Rodrigues et al. 2014). Unfortunately, no stars in this sample matched APOKASC stars with distance measurements, but we include it in the pipeline in hopes that future versions of the APOKASC catalog will overlap with future versions of this catalog. Our second choice, if the star is a RC star, is to use distances derived from the APOGEE RC catalog. These distances are cited to have $5 \%-10 \%$ random errors, and 71 stars of the 907 run through the apOrbit pipeline are RC stars. If the star has neither of the above distances available, we adopt the spectrophotometic distance estimates derived by Santiago et al. (2015), Hayden et al. (2015), or Schultheis et al. (2014), based on which estimate has the lowest error. These distances generally have $<15 \%-20 \%$ uncertainties, and for most of the RG stars run through the apOrbit pipeline (489 stars), we adopt these distances. The six PMS stars in this sample are located in the young cluster IC 348 ( $d=316 \pm 22$ pc; Herbig 1998), so we adopt the distance to this cluster as the approximate distance to these stars. From the adopted distance, $d$, we estimate the luminosity of the star, and thus its radius and mass:

$$
\begin{gathered}
M_{\mathrm{bol}}=m_{\mathrm{bol}}-5 \log (d)+5 \\
L_{\star}=10^{-0.4\left(M_{\mathrm{bol}}-4.77\right)} L_{\odot} \\
R_{\star}=\sqrt{\frac{L_{\star}}{4 \pi \sigma_{\mathrm{SB}} T_{\mathrm{eff}}^{4}}}
\end{gathered}
$$




$$
M_{\star}=\frac{10^{\log g} R_{\star}^{2}}{G} .
$$

Following typical propagation of uncertainties, these techniques produce a mass uncertainty floor of $26 \%$ due to the uncertainty in $\log g$. The median of mass uncertainties for these techniques is around $28 \%$.

If a giant star has no distance measurement available, we adopt a characteristic mass from a TRILEGAL (Girardi et al. 2005) simulation using parameters typical of APOGEE giants. The median mass for all stars in this simulation with $\log g<3.8$ and $3500 \mathrm{~K}<T_{\text {eff }}<5000 \mathrm{~K}$ in the direction of Galactic Coordinates $(\ell, b)=(0,40)$ is $M_{\star}=1.6 \pm 0.6 M_{\odot}$ ( $\sim 40 \%$ mass uncertainty), which we adopt as the typical mass for all giant stars without a distance measurement. From this we derive $R_{\star}=\left(G M_{\star} / 10^{\log g}\right)^{1 / 2}$ and $d$, as for the dwarfs, both with typical estimated uncertainties of $25 \%$. Fortunately, we only need to adopt this type of mass estimate for one star run through the aporbit pipeline.

\subsection{Keplerian Orbit Fitting}

Once a star has mass and radius estimates, we can attempt to search for periodic signals and derive Keplerian orbits from its RV measurements. Only stars with at least eight "good" visits have enough degrees of freedom to attempt the six and seven parameter Keplerian orbit fits. For each star meeting this criterion, we attempt orbital fits with and without a long-term underlying linear trend. The linear fit accounts for additional long-term RV variability that may be indicative of an additional companion with a period longer than we can detect reliably, or long-term instrumental effects.

\subsubsection{Period-finding and Selection of Initial Conditions}

We employ the Fast $\chi^{2}$ Period Search $\left(F \chi^{2}\right)$ algorithm (Palmer 2009) to search for periodic signals. This algorithm chooses the period based on the largest reduction in $\chi^{2}$ between a sinusoidal fit employing the first $n_{h}$ harmonics of a fundamental period, $p_{i}$, compared to a global $n_{d}$-degree polynomal fit. The $F \chi^{2}$ algorithm uses harmonics of the fundamental period in its fits, which produces improved performance with non-circular orbits compared to the traditional Lomb-Scargle algorithm (Scargle 1982). Another advantage of the $F \chi^{2}$ algorithm is a built-in avoidance of periodic signals introduced by the cadence of the data, i.e., inputting data taken every $n$ days will not return a $n$-day period as the best fit.

For our purposes, we employ three harmonics $\left(n_{H}=3\right)$, execute a search in four (logarithmic) period bins ( $0.3-3$ days, 3-30 days, 30-300 days, and 300-3000 days), and oversample ten times the default frequency sampling such that the frequency step is $\Delta f=1 /\left(10 n_{h} \Delta T\right)$, where $\Delta T$ is the longest temporal baseline of the observations. The search is executed once with a constant $\left(n_{d}=0\right)$ fit and once with a linear fit $\left(n_{d}=1\right)$. The periods in each bin, $p_{j}$ that produce the greatest reduction in $\chi^{2}, \Delta \chi_{\max }^{2}$, are then assessed for their significance using the following criterion:

$$
P_{n-2 n_{h}}\left(\Delta \chi_{\max }^{2}\right) \geqslant 0.997,
$$

where $P_{n-2 n_{h}}\left(\Delta \chi_{\max }^{2}\right)$ is the probability for a $\chi^{2}$ distribution with $n-2 n_{h}$ degrees of freedom, and $n$ is the number of RV epochs. The above limit is the equivalent of a $3 \sigma$ detection. Periods that are not deemed significant by this metric are not used for full Keplerian orbit fitting. The significant periods $\left(p_{j}\right)$ and their harmonics $(1 / 3,1 / 2,2$, and 3 times each value of $p_{j}$ ) are then each used for Keplerian orbit fitting.

\subsubsection{Derivation of Keplerian Orbits}

Once the best periods are identified, Keplerian models with those periods are fit to the RV measurements using the MPFIT algorithm (Markwardt 2009). MPFIT is a Levenberg-Marquardt nonlinear least squares fitter implemented in IDL. This code is wrapped in an IDL code MP_RVFIT used in the MARVELS survey (De Lee et al. 2013). MP_RVFIT takes the input period and searches parameter space of the other Keplerian orbital parameters $\left(K, e, \Omega, T_{p}\right.$, and global velocity trends) and returns the Keplerian model that satisfies the period with the lowest $\chi^{2}$.

Having a precise period is extremely important for acquiring an accurate Keplerian model, and simply submitting the periods from the period-finding algorithm to MP_RVFIT often leads to unsatisfactory results. Here we describe the bisector method implemented to achieve the best possible period. We initially submit the periods described above to MP_RVFIT, and keep the three periods $\left(p_{k, 0}\right)$ that produce the best fits based on the modified reduced-chi-squared goodness of fit statistic, $\chi_{\text {mod }}^{2}$, described in Section 3.3.4. For each of these periods we implement a bisector method to narrow in on the exact period. For each $p_{k}$ we run MP_RVFIT with three periods: $p_{k, 0}$ and $p_{k, 0} \pm \Delta p_{0}$, where $\Delta p_{0}=0.5 p_{k, 0}$. We then compare the $\chi_{\bmod }^{2}$ for the best fits for the three periods, and update $p_{k}$ and $\Delta p$ accordingly:

$$
\begin{gathered}
\text { If } \chi_{p_{k, i}}^{2} \leqslant \chi_{p_{k, i} \pm \Delta p_{i}}^{2}: \\
p_{k, i+1}=p_{k, i}, \Delta p_{i+1}=\Delta p_{i} / 2, \\
\text { If } \chi_{p_{k, i} \pm \Delta p_{i}}^{2}<\chi_{p_{k, i}}^{2}: \\
p_{k, i+1}=p_{k, i} \pm \Delta p_{i}, \Delta p_{i+1}=\Delta p_{i} .
\end{gathered}
$$

For the $\chi_{p_{i}-\Delta p_{i}}^{2}<\chi_{p_{i}}^{2}$ case, if $p_{i}-2 \Delta p_{i}<0.1$, then we use $\Delta p_{i}=\Delta p_{i} / 2$ for the next update. This iteration is performed until the change in $\chi_{\text {mod }}^{2}$ is less than 0.01 or $n_{\text {iter }}=50$ iterations are reached. The distribution of the required number of iterations for systems in the final sample had a median of 15 with few systems above 25 . Therefore, the choice to terminate systems on their 50th iteration is more than justified as these systems are unlikely to converge in a timely manner. These systems are also not included in the final catalog (see Section 4.2). The final values of $p_{k, n_{\text {iter }}}$ are then submitted to MP_RVFIT one final time, and the results saved for the catalog. The data saved are described in Section 4. For a few example Keplerian orbit models see Figure 5. 

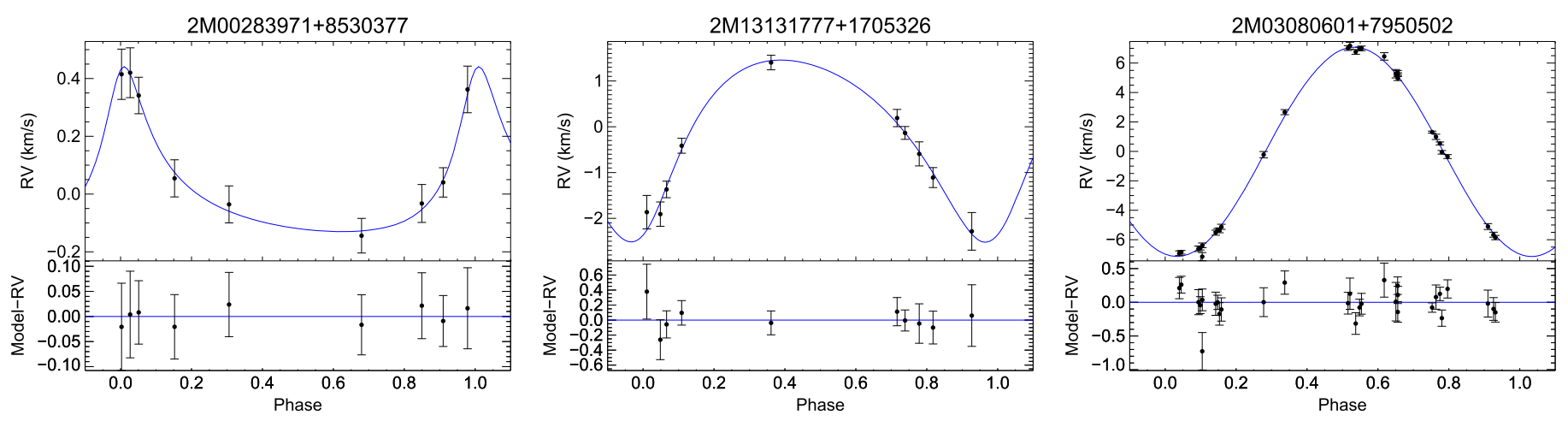

Figure 5. RV curves for a few example systems. In each plot, the top panel presents the phased RV measurements with a line showing the best fit model and the bottom panel shows the residuals of the fit. Similar figures are available online for every star in the gold sample (see Appendix B). Left Panel: a planetary-mass ( $m \sin i=4.60 M_{\mathrm{Jup}}$ ) companion in a $P=41.3$ day, $a=0.25 \mathrm{AU}$ orbit with $e=0.566$, and $K=0.29 \mathrm{~km} \mathrm{~s}^{-1}$. This orbit has uniformity index (see Section 3.3.4) values of $U_{N}=0.886$ and $V_{N}=0.737$. Middle Panel: a BD-mass companion $\left(m \sin i=22.6 M_{\mathrm{Jup}}\right)$ companion in a $P=24.3$ day, $a=0.15$ AU orbit with $e=0.293$, $K=1.99 \mathrm{~km} \mathrm{~s}^{-1}$. This orbit has uniformity index values of $U_{N}=0.871$ and $V_{N}=0.935$. Right Panel: binary system with a $m \sin i \approx 0.304 M_{\odot}$ secondary in a $P=184$ day, $a=0.68$ AU orbit with $e=0.004, K=7.11 \mathrm{~km} \mathrm{~s}^{-1}$. This orbit has uniformity index values of $U_{N}=0.937$ and $V_{N}=0.869$.

\subsubsection{From Orbital to Physical Parameter Estimates}

Directly from the orbital parameters, we can calculate the projected semimajor axis of the primary star:

$$
a_{\star} \sin i=\frac{\mathrm{KP}}{2 \pi} \sqrt{1-e^{2}}
$$

From this measurement we can define the mass function of the system:

$$
f\left(m, M_{\star}\right)=4 \pi^{2} \frac{\left(a_{\star} \sin i\right)^{3}}{\mathrm{GP}^{2}}=\frac{(m \sin i)^{3}}{\left(M_{\star}+m\right)^{2}} .
$$

This quantity is saved in the catalog, but we also attempt to estimate the secondary mass directly:

$$
m \sin i=\left[f\left(m, M_{\star}\right) M_{\star}^{2}\left(1+\left(m / M_{\star}\right)\right)^{2}\right]^{1 / 3} .
$$

The general case of this equation cannot be solved analytically, but often when dealing with planetary companions, we can make the assumption that $m \ll M_{\star}$, and thus can make the approximation $m \sin i \approx\left(f\left(m, M_{\star}\right) M_{\star}^{2}\right)^{1 / 3}$. For companions with $m \sin i<0.1 M_{\star}$, this approximation is accurate to within $10 \%$, but this sample contains higher-mass companions for which we want reasonable mass estimates. In these cases we solve the above equation iteratively, initially assuming $m=0$, returning the above estimate, and iterating until $m \sin i$ changes by $<10^{-4} M_{\odot}$. Since we are interested in estimating the minimum mass of the companion, we solve for the $\sin i=1$ case, and thus use $m \approx m \sin i$ after the first iteration. This iterative method for determining $m$ was tested for a variety of mass ratios and a variety of starting points for $m$ (not just $m=0$ ). From these tests, we have found this method to be quite robust.

Finally, from the estimate of $m \sin i$, we provide an estimate of the semimajor axis, $a$, of the secondary:

$$
a=a_{\star} \sin i \frac{M_{\star}}{m \sin i} .
$$

\subsubsection{Quality Control and Selection of Best Fits}

Finally we compile the three best models from the run with no global linear fit and the three best models from the linear fit run, and compare them to select the best overall fit. Ideally the phase and velocity coverage of the model are uniformly sampled by the data, and we aimed to preferably select models that are as close to this ideal as possible. A useful way to quantify the phase coverage of the data is the uniformity index (Madore \& Freedman 2005):

$$
U_{N}=\frac{N}{N-1}\left[1-\sum_{i=1}^{N}\left(\phi_{i+1}-\phi_{i}\right)^{2}\right]
$$

where the values $\phi_{i}$ are the sorted phases associated with the corresponding Modified Julian Date (MJD) of the measurement $i$, and $\phi_{N+1}=\phi_{1}+1$. This statistic is normalized such that $0 \leqslant U_{N} \leqslant 1$, where $U_{N}=1$ would indicate a curve evenly sampled in phase space. Using a similar derivation, we also define an analogous "velocity" uniformity index with the same properties as $U_{N}$ :

$$
V_{N}=\frac{N}{N-1}\left[1-\sum_{i=1}^{N}\left(\nu_{i+1}-\nu_{i}\right)^{2}\right] .
$$

We define a "velocity phase," $\nu_{i}=\left(v_{i}-v_{\min }\right) /\left(v_{\max }-v_{\min }\right)$, to have the same properties as $\phi_{i}$ above, where the values $\nu=0$ and $\nu=1$ indicate the minimum and maximum velocities of the model, $v_{\min }$ and $v_{\max }$. The values of $v_{i}$ are the RV measurements, sorted by their value, with the adopted global velocity trend subtracted. For models that do not apply a global linear trend, the trend subtracted is the average of the raw velocities: $v_{i}=v_{\text {raw }, i}-\bar{v}_{\text {raw }}$. Measured velocities below the minimum or above the maximum are assigned $\nu=0$ and $\nu=1$, respectively. The purpose of this metric is to prevent the pipeline from selecting an extremely eccentric orbit when the data do not support such a model. Values of $U_{N}$ and $V_{N}$ are given in the example RV curves of Figure 5.

Combining the above statistic with the traditional reduced $\chi^{2}$ goodness-of-fit statistic $\left(\chi_{\text {red }}^{2}\right)$, we define the modified $\chi^{2}$ statistic,

$$
\chi_{\mathrm{mod}}^{2}=\frac{\chi_{\mathrm{red}}^{2}}{\sqrt{U_{N} V_{N}}}
$$


by which the models are ranked. In the case that $U_{N}=0$ or $V_{N}=0, \chi_{\text {mod }}^{2}$ would be recorded as a floating-point infinity and automatically be ranked below all other fits. However, there are some conditions where the fit is unacceptable, but still may be selected as the best fit using the above metric. Therefore, we defined criteria that split the fits into "good" and "marginal" fits. Any of the following criteria would warrant a "marginal" classification:

1. Periods within $5 \%$ of $3,2,1,1 / 2$, or $1 / 3$ day,

2. Periods, $P$, longer than twice the baseline, $2 \Delta T$,

3 . Extremely eccentric solutions $(e>0.934)^{26}$,

4. Orbital solutions that send the companion into the host star: $a(1-e)<R_{\star}$,

5. Poor phase and velocity coverage $\left(U_{N} V_{N}<0.5\right)$.

The good and marginal fits are ranked by $\chi_{\text {mod }}^{2}$ separately, and the best fit is the good fit with the lowest $\chi_{\text {mod }}^{2}$. If all of the fits were deemed marginal, then the best fit is the marginal fit with the lowest $\chi_{\bmod }^{2}$. For more details on the verification and performance of the apOrbit pipeline, see Appendix A.

\section{BUILDING THE APOGEE CANDIDATE COMPANION CATALOG}

A total of 907 stars were successfully run through the aporbit pipeline. Of these, the $F \chi^{2}$ algorithm found significant periodic signals for 749 , which were submitted for full Keplerian orbit fitting. In this section, we describe the data available for these stars, and the selection of companion candidates from the best Keplerian orbit fit to these stars. Information on catalog content and access can be found in Appendix B.

\subsection{Selecting Statistically Significant Astrophysical RV Variations}

In many cases, the RV variations are within the measurement errors, so the derived semi-amplitude for the orbit may be masked by measurement error. In these cases, we cannot reliably state that the RV variations are astrophysical in nature. However, even astrophysical RV variations may not be due to the presence of a companion. Many stars, especially giant stars, which compose a large part of this sample, can have high levels of intrinsic RV variability. To estimate this stellar RV jitter, we adopted the relation found by Hekker et al. (2008):

$$
v_{\text {jitter }}=2(0.015)^{\frac{1}{3} \log g} \mathrm{~km} \mathrm{~s}^{-1},
$$

where, again, $\log g$ is the logarithm of the surface gravity in $\operatorname{cgs}$ units. We define a total RV uncertainty for each point in the model fit by combining this quantity with the RV measurement uncertainties, $\sigma_{v}$ :

$$
v_{\mathrm{unc}}=\sqrt{\sigma_{v}^{2}+v_{\mathrm{jitter}}^{2}} .
$$

We use the following criteria to select statistically significant companion candidates:

$$
\frac{K}{\tilde{v}_{\text {unc }}} \geqslant 3+3\left(1-V_{N}\right) e,
$$

\footnotetext{
${ }^{26}$ This is the eccentricity of HD $80606 \mathrm{~b}$, the largest eccentricity in the exoplanets.org database.
}

where $\tilde{v}_{\text {unc }}$ is the median RV uncertainty of the model fit, $K$ is the RV semi-amplitude of the best-fit model for the star, and $V_{N}$ is the velocity uniformity index described in Section 3.3.4. We include the $\left(1-V_{N}\right) e$ term to increase the significance criteria for eccentric systems, particularly those that have poor velocity coverage. Thus, a perfectly covered eccentric orbit $\left(V_{N}=1\right)$ would be treated the same as a circular orbit $(e=0)$. Using these criteria, 698 stars are selected as statistically significant companion candidates.

\subsection{Refining the Catalog: Defining The Gold Sample}

In an effort to minimize the number of false positives in this sample and reduce the number of systems with incorrectly derived orbital parameters (see Appendix A), we eliminate candidates that do not satisfy the following criteria:

1. None of "marginal fit" criteria described in Section 3.3.4 are met.

2. The Keplerian fits must be reasonably good, which we quantify as the criteria:

$$
\begin{gathered}
\frac{K}{|\Delta \widetilde{v}|} \geqslant 3+3\left(1-V_{N}\right) e, \\
\chi_{\text {mod }}^{2} \leqslant \frac{K /|\Delta \widetilde{v}|}{3+3\left(1-V_{N}\right) e}, \\
\chi_{\text {mod }}^{2} \leqslant \frac{K / \widetilde{\text { unc }}_{\text {unc }}}{3+3\left(1-V_{N}\right) e},
\end{gathered}
$$

where $|\Delta \widetilde{v}|$ is the median absolute residuals of model fit. From simulations and visual inspection of orbits, orbits with large median $K / v_{\text {unc }}$ or $K / \Delta v$ reproduced the correct parameters and had reasonable fits at much larger values of $\chi_{\bmod }^{2}$ than orbits with lower values. A major exception to this trend were large $K / \widetilde{v}_{\text {unc }}$ orbits due to high $e$ or orbits with poor velocity sampling (low $V_{N}$ ), so the metric above includes terms to penalize fits with high eccentricity $\left(1-e\right.$ term) or low $V_{N}$ (which inflates $\left.\chi_{\bmod }^{2}\right)$ Therefore this "good fit" limit is stricter for such systems by employing the $\chi_{\text {mod }}^{2}$ metric discussed above. Previous cuts also guaranteed that no systems with $\chi_{\text {mod }}^{2} \leqslant 1$ are excluded because of this metric.

3. The best fit must not require the maximum number of period iterations to converge; as described in Section 3.3.2. Systems that reach that maximum limit of iterations in the fitter did not converge on a solution, and the orbital parameters output are likely to be unreliable.

As mentioned above, many of these criteria were inspired by the testing of simulated systems with known orbital parameters described in Appendix A. Using these refined criteria, 382 stars (55\% of the statistically significant RV variable sample) were selected to be a part of the "gold sample," which represent the best-quality companion candidates detected by APOGEE. This is not to say that the other $45 \%$ of the statistically significant $\mathrm{RV}$ variable sample do not have companions, and there very well may be accurately reproduced companions from the nongold sample. However, the likelihood of either false positives or poorly characterized systems is much higher for the nongold sample than for the gold sample, hence we only present the 382 stars in the gold sample here. 
Table 1

A Census of APOGEE Gold Sample Companion Candidates

\begin{tabular}{lcccc}
\hline \hline Population & Binaries $^{\mathrm{a}}$ & BDs $^{\mathrm{b}}$ & Planets $^{\mathrm{c}}$ & Total \\
\hline Host Star Classification $^{\mathrm{d}}$ & & 5 & 0 & 23 \\
Red Clump (RC) & 18 & 56 & 9 & 180 \\
Red Giant (RG) & 115 & 10 & 3 & 22 \\
Subgiant (SG) & 9 & 41 & 45 & 157 \\
Dwarf (MS) & 71 & 1 & 0 & 2 \\
PMS & 1 & 36 & 13 & 119 \\
Host Star Metallicity & 70 & 62 & 42 & 222 \\
[Fe/H] $\geqslant 0$ & 118 & 14 & 2 & 41 \\
$-0.5 \leqslant[$ Fe/H] $<$ & 25 & & & \\
[Fe/H] $<-0.5$ & 180 & 91 & 56 & 327 \\
Galactic Environment & & 18 & 1 & 50 \\
Thin Disk & 31 & 3 & 0 & 5 \\
Thick Disk & 2 & 112 & 57 & 382 \\
Halo & 213 & & & \\
\hline Catalog Totals & & & & \\
\hline
\end{tabular}

Notes.

${ }^{\mathrm{a}}$ We define likely stellar-mass binaries as having a companion with $\langle m\rangle>0.08 M_{\odot}$.

${ }^{\mathrm{b}}$ Brown dwarf companions: $0.013 M_{\odot}<\langle m\rangle \leqslant 0.08 M_{\odot}$.

${ }^{c}$ Planetary-mass companions: $\langle m\rangle \leqslant 0.013 M_{\odot}$.

${ }^{\mathrm{d}}$ Host star classification and abbreviations discussed in Section 3.2.1.

e To truly distinguish between Thin and Thick Disk populations, a full analysis of the chemistry and kinematics of the stars would be needed. Here we simply present a census of companion as a function of height above the midplane, and use these criteria: Thin Disk $=|Z|<1 \mathrm{kpc}$, Thick Disk $=1 \mathrm{kpc} \leqslant|Z|<5 \mathrm{kpc}$, Halo $=|Z| \geqslant 5 \mathrm{kpc}$.

\section{CENSUS OF GOLD SAMPLE COMPANION CANDIDATES AND DISCUSSION OF INITIAL RESULTS}

In this section, we present a census of the 382 companion candidates in the catalog. Of these, 376 are newly discovered small separation companion candidates. Table 1 provides a broad overview of the distributions of the companion candidates in terms of companion type (planet, BD or binary), host star type (e.g., giant versus dwarf), and approximate Galactic environment (disk versus halo). We discuss each of these distributions and their implications in more detail in the subsections below. From this point on, we use $\langle m\rangle$ to indicate the maximum-likelihood value of the companion mass, $m$, based on the expectation value of $i$, defined as $\langle\sin i\rangle=\int_{0}^{\pi / 2} P(i) \sin i d i=\int_{0}^{\pi / 2} \sin ^{2} i d i=\pi / 4$. Therefore, $\langle m\rangle=(4 / \pi) m \sin i$, and we use this number to differentiate between companion types to account for inclination effects in a statistical manner.

\subsection{Orbital Distribution of Companion Candidates}

The top panel of Figure 6 presents the overall distribution of $\langle m\rangle$ and orbital semimajor axis of the candidate companions in the gold sample. In this figure, there appears to be two distinct companion mass regimes in which the candidates lie, and thus suggests different companion formation channels. The upper regime is the binary star track, where the companion likely formed with (or shortly after) the primary from fragmentation of the cloud or disk from which the primary formed. The lower regime is the "planet" track, where the companion likely formed after the primary either through core accretion or gravitation instability in the disk surrounding the protostar. The trend of the lower planetary boundary mimics the sensitivity of the APOGEE survey (see Equation (32) with $\tilde{\sigma}_{v}=100 \mathrm{~m} \mathrm{~s}^{-1}$ ). However, the trend of the planet track's upper boundary cannot be explained by a selection or sensitivity effect. One interpretation of the gap between the two regimes is a manifestation of the BD desert in the data, but the two tracks appear to merge at larger semimajor axes ( $a>0.1-0.2 \mathrm{AU})$. The implications of this are discussed below.

\subsubsection{Combing the BD Desert}

The top panel of Figure 6 indicates that this sample reproduces the $\mathrm{BD}$ desert, but only for orbits with $a<$ $0.1-0.2$ AU ( $P<10-30$ days), which is significantly less than the 3 AU extent of the desert as stated in Grether \& Lineweaver (2006). However, their sample mostly considered solar-like dwarf hosts, while this sample contains stars with a variety of spectral types, as well as many evolved stars. From the top panel of Figure 7, it appears that the relative number of BD companions decreases as host mass increases for MS hosts. Likely M dwarfs (MS with $M_{\star}<0.6 M_{\odot}$ ) have roughly equal numbers of $\mathrm{BD}$ and stellar-mass companions, while $\mathrm{K}$ dwarfs (MS with $0.6<M_{\star} / M_{\odot}<0.85$ ) have roughly half the number of BD candidate companions as stellar-mass candidate companions. The $\mathrm{G}$ dwarfs (MS with $0.85<M_{\star} / M_{\odot}<1.1$ ) show a similar relative number of BD companions compared to stellar-mass companions, but they are less uniformly distributed throughout the $\mathrm{BD}$ mass regime compared to the lower mass BD candidate hosts, suggesting a higher probability that many of these $\mathrm{BD}$ candidates are scattered into the BD mass regime by inclination effects. These results leads one to believe the interpretation of Duchêne \& Kraus (2013) that the BD desert is simply a special case for solar-mass stars of a more general lack of extreme mass ratio $(q \lesssim 0.1)$ systems. For example, if, in general, systems with $q<0.08$ are rare (i.e., a $\mathrm{BD}$ companion around a $1 M_{\odot}$ companion), then a relatively high-mass $\mathrm{BD}$ companion $\left(m>0.04 M_{\odot}\right)$ orbiting a $0.5 M_{\odot}$ star should be a more common occurrence.

Out of the 112 BD companion candidates in this sample, 71 orbit evolved stars. All but two of the giant (RC and RG) hosts have masses $>0.8 M_{\odot}$ and only one of the SG hosts has a mass $<1 M_{\odot}$. Considering that stars like the Sun lose up to a third of their mass on the red giant branch (RGB), it is a reasonable assumption that a vast majority of the evolved stars in this sample descended from main-sequence F (or earlier) dwarfs. As can be seen from the bottom panel of Figure 7, the evolved stars have roughly half the number of BD candidate companions as stellar-mass candidate companions, and the BD-mass candidates are distributed throughout the BD-mass regime, similar to the $\mathrm{K}$ dwarf distribution. If the evolved stars are indeed evolved $\mathrm{F}$ dwarfs, and we follow the progression from above, one would expect these stars to have a smaller relative number of $\mathrm{BD}$ companions compared to even the $\mathrm{G}$ dwarfs. However, it has been previously suggested that the BD desert observed for Solar-like stars may cease to exist for $\mathrm{F}$ dwarf stars (Guillot et al. 2014). Their proposed explanation of this effect is that $G$ dwarfs are more efficient at tidal dissipation. In general, compared to Jupiter-mass planets, more massive small separation companions undergo stronger tidal interaction with their host star through angular momentum exchange. Stellar-mass companions, however, have sufficient orbital angular momentum to remain in a stable orbit, which explains the demise of small separation BD-mass but not 

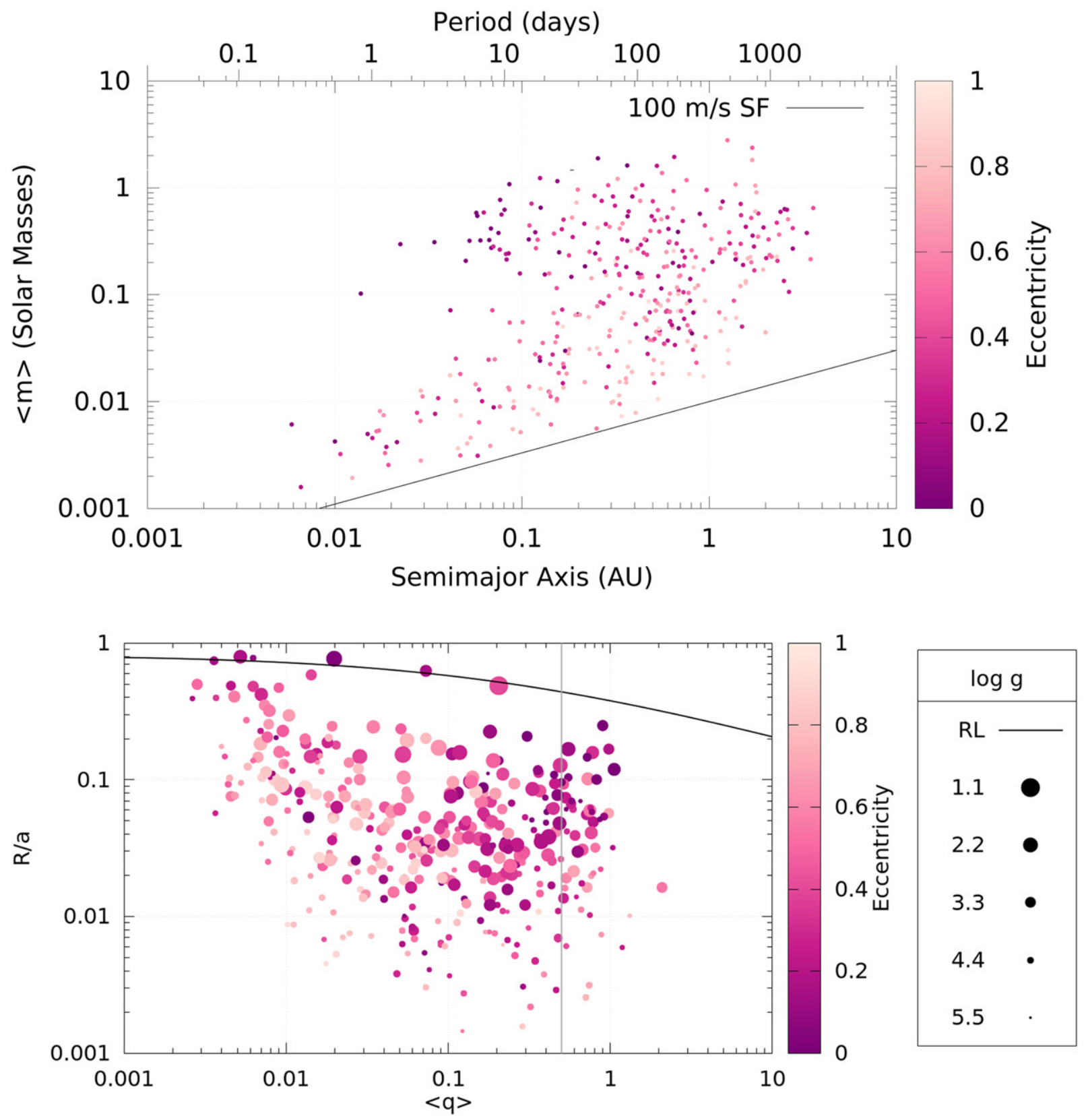

Figure 6. Top Panel: orbital distribution of companion candidates in the 382-star gold sample with orbital semimajor axis (a) in AU on the abscissa and maximumlikelihood companion mass $(\langle m\rangle=(4 / \pi) m \sin i)$ in $M_{\odot}$ on the ordinate. The top horizontal axis gives the approximate period for the companion in days as well. Color represents the orbital eccentricity of the companion, with dark magenta representing circular orbits. The black line is the sensitivity function (SF; Equation (32)) for $100 \mathrm{~m} \mathrm{~s}^{-1} \mathrm{RV}$ precision. Systems below this line would generally be undetectable by APOGEE. Bottom Panel: orbital distribution of companion candidates with $\langle q\rangle=\langle m\rangle / M_{\star}$ on the abscissa, and $R_{\star} / a$ on the ordinate. Color again represents eccentricity, and point size indicates the surface gravity (log $g$ ) of the host. The gray vertical line marks systems with $\langle q\rangle>0.5$, and the black line across the top of the panel indicates the Roche limit (RL; Equation (31)) of the host star.

stellar-mass companions. However, F (and earlier) dwarfs are known to remain rapid rotators $\left(v_{\text {rot }} \sim 20-100 \mathrm{~km} \mathrm{~s}^{-1}\right)$ throughout their main-sequence lifetimes due to their smaller outer convective zones leading to weaker magnetic breaking. This means $\mathrm{F}$ dwarfs are also less efficient at extracting angular momentum from an orbiting companion. Therefore, rapid rotators such as $\mathrm{F}$ dwarfs inhibit tidal dissipation, which explains this " $F$ dwarf oasis" for BD companions. The dynamical model presented in Figure 4 of Guillot et al. (2014) shows that a companion in the BD-mass regime on an initial 3 day orbit around a $1 M_{\odot}$ star will survive for $<40 \%$ of the star's main sequence lifetime $(\lesssim 4 \mathrm{Gyr})$, while the same companion around a host star with $M_{\star}>1.2 M_{\odot}$ will survive for at least the entirety of the host star's main sequence lifetime ( $6.5 \mathrm{Gyr}$ for a $1.2 M_{\odot}$ star). The presence of a large number BD companions orbiting the evolved stars in this sample strongly supports this "F dwarf oasis" hypothesis.

However, the tidal effects explanation would only strongly affect the closest-in companions. Since the rotation period of a G dwarf is $P_{\star}=30$ days (compared to a few days for an F dwarf), tidal dissipation could only explain BD companions with orbital periods less than 30 days, and the majority of the BD candidate companions in this sample have periods significantly greater than that. Therefore, tidal dissipation can only explain the BDs (or lack thereof) with orbits within 0.2 AU. Curiously, this sample reproduces the BD desert out to 

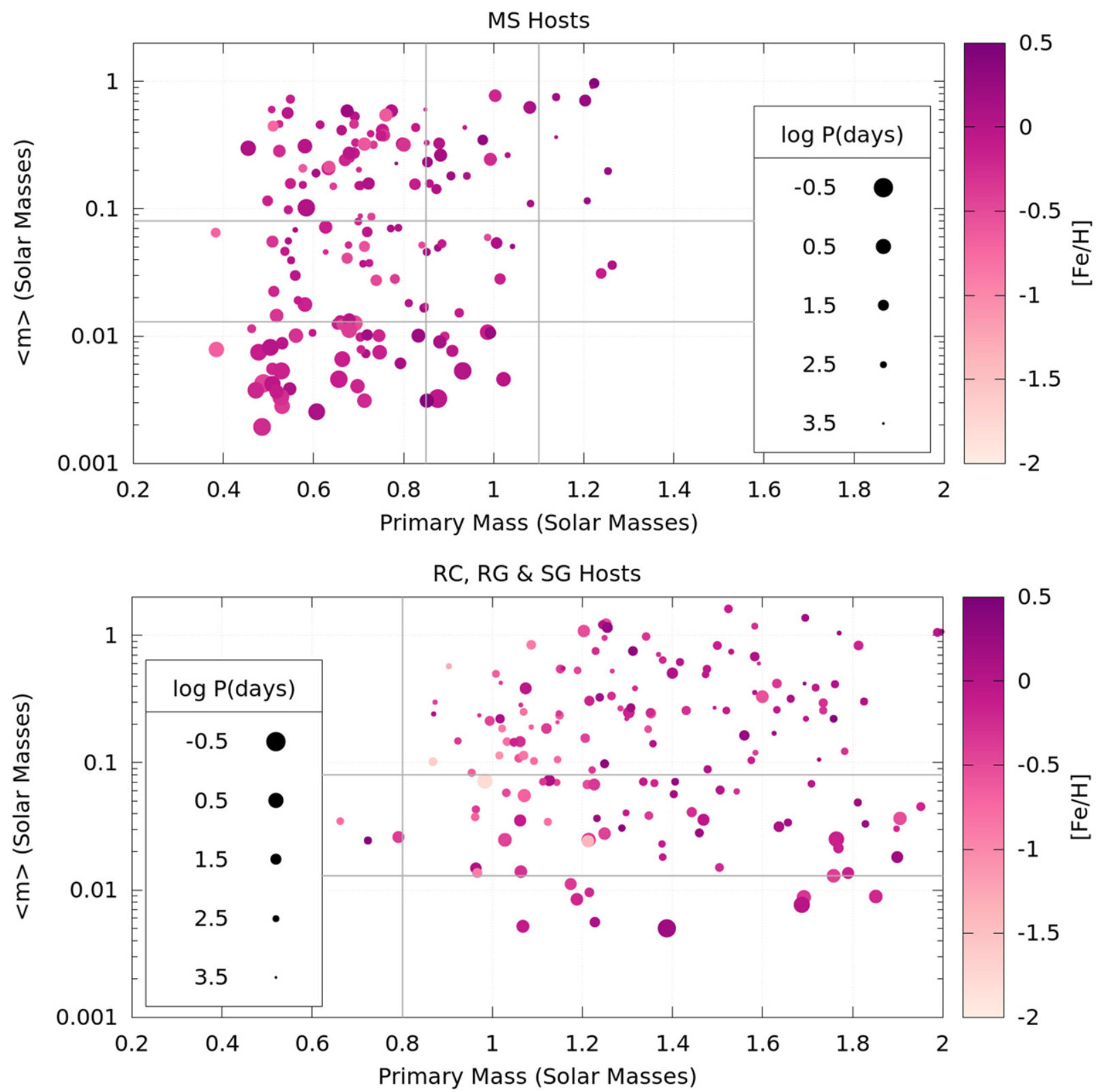

Figure 7. Modification of Figure 3 from Guillot et al. (2014), with $M_{\star}$ on the abscissa and maximum-likelihood companion-mass $(\langle m\rangle)$ on the ordinate. Color represents the host stars' metallicity, and point size represents the period of the companion in log days. Larger points here indicate companions that are more likely to be undergoing tidal interaction with their host star. Top Panel: stars in the gold sample selected as MS stars. The vertical lines mark nominal G dwarfs $\left(0.85<M_{\star} / M_{\odot}<1.1\right)$, and the horizontal lines mark the BD mass regime $\left(0.013<\langle m\rangle / M_{\odot}<0.08\right)$. Bottom Panel: remaining stars in the gold sample with $M_{\star}<2 M_{\odot}$. The horizontal lines again mark the BD mass regime, and the vertical line marks $M_{\star}=0.8 M_{\odot}$. It would be a reasonable expectation that a giant star above this mass evolved from a star earlier than a G dwarf since solar-like stars loose about one third of their mass on the RGB.

approximately $0.2 \mathrm{AU}$, suggesting this mechanism may indeed play a role in shaping the BD desert. Another possible explanation for the presence of $\mathrm{BD}$ candidate companions is Roche lobe overflow of the star as it evolves off the main sequence onto an orbiting planetary-mass candidate, allowing it to grow to BD mass as the star evolves up the RGB. Eggleton (1983) gives the following approximation for the Roche lobe of a primary donor star with mass $M_{1}$ orbited by a companion with $M_{2}$ :

$$
\frac{r_{1}}{a}=\frac{0.49 q^{-2 / 3}}{0.6 q^{-2 / 3}+\ln \left(1+q^{-1 / 3}\right)},
$$

where, $q=M_{2} / M_{1}, a$ is the separation of the two bodies, and $r_{1}$ is the Roche lobe radius of the potential donor. In the bottom panel of Figure 6, we mark the Roche lobe as a function of $\langle q\rangle$. As an interesting note, it appears there are seven stars in this sample that are currently at or near Roche lobe overflow, three of which are currently of planetary mass, and three of which are BD mass. These systems will all be the subject of further scrutiny. In general, for a 1-10 $M_{\text {Jup }}$ planet to cause a $\sim 1 M_{\odot}$ primary to overflow its Roche lobe, the radius of the primary would have to exceed $\sim 70 \%-80 \%$ of the separation between the two bodies. This would not be an unreasonable expectation for a companion orbiting within $1 \mathrm{AU}$, as solar-mass stars can 
achieve radii approaching $1 \mathrm{AU}$ at the tip of the RGB. Therefore, this mechanism may be a way to explain the relatively large number of BD companion candidates orbiting the evolved stars in this sample. Overall, this catalog's large number of systems with short-period BD companion candidates challenges the notion of the BD desert as we know it, and certainly warrants further investigation.

\subsubsection{Eccentricity Distribution}

In Figure 6, we also see the distribution of orbital eccentricities. As expected, the smallest-separation $(a<0.1$ AU) stellar-mass companions all have circular orbits. The circularization cutoff period increases with the age of the system with 5-10 Gyr systems having cutoff periods of 12-20 days (Mathieu et al. 2004). All of the binary companions in this catalog with $a<0.1$ AU have $P<20$ days. Therefore, the distribution of eccentricities for the binary systems in this sample, with circular orbits at small separation, and eccentric orbits a large separations is not unexpected. The closest $(a<0.01 \mathrm{AU})$ planetary-mass companions appear to have also circularized, as expected, but a surprising result is the relatively large fraction of eccentric orbits for relatively close-in planetary-mass candidate companions. For the RG and RC hosts, one interpretation of these eccentricities is ongoing tidally induced migration (see Section 5.2.1 for further discussion of this). However, the majority of the smallseparation planetary and BD candidate companions orbit dwarf and SG stars. For these systems, their higher eccentricities may be further evidence for the mechanism suggested by Tsang et al. (2014) whereby stellar illumination heating a gap cleared by a forming planet may excite the eccentricity of the planet in the gap.

\subsubsection{High Mass Ratio Systems}

Of this catalog's candidate companion systems, there are 50 systems with a mass ratio $\langle q\rangle=\langle m\rangle / M_{\star} \geqslant 0.5$ (see bottom panel of Figure 6). One would expect that these systems would manifest themselves as SB2s, but these systems show no strong indication of such behavior in their APOGEE spectra. Of these, 24 are RG stars, which would explain their lack of SB2 behavior, as their companion is likely still on the main sequence, and thus the flux ratio would be too large. However, this still leaves $26 \mathrm{MS}$ and SG hosts, of which one explanation is that they host massive compact objects, such as stellar remnants. These seven companions have $0.3 M_{\odot}<\langle m\rangle<1.2 M_{\odot}$, which would indicate these systems might host white dwarf companions, eight of which may be low-mass $\left(\langle m\rangle<0.45 M_{\odot}\right)$ He-core white dwarfs (Liebert et al. 2005). Furthermore, two of the systems with a RG host have $\langle q\rangle>1$, indicating the companion has already completed its evolution, and the recovered $\langle m\rangle$ of the companions (2.8 and $1.6 M_{\odot}$ ) indicates they may be neutron stars.

\subsection{Host Star Distribution}

Solar type stars (i.e., $\mathrm{G}$ dwarfs) have been the primary focus of exoplanet and stellar multiplicity studies. Out of the 382 stars in this sample, only 36 are solar-type (MS with $5000 \mathrm{~K}<T_{\text {eff }}<6000 \mathrm{~K}$ ) stars. Figure 8 reveals that, in addition to the solar-type stars, this sample contains cool dwarfs, subgiant and giant stars, which allows us to probe many different stellar types and stages of stellar evolution. Figure 8 also presents distributions of the stellar parameters of the host stars in this sample.

\subsubsection{The Fate of Companions: Exploring Evolved Host Stars}

Tidal dissipation is thought to play an important role in the destruction of planetary systems as a star evolves off the main sequence and expands (Penev et al. 2012). This sample contains $225 a<3$ AU candidate companions to evolved stars, indicating either many initial small separation companions survive engulfment or farther-orbiting planets undergo increasing tidal migration as its host ascends the giant branch, bringing the companion closer to its host star. The nine candidate planetary-mass $\left(\langle m\rangle<0.013 M_{\odot}\right)$ companions orbiting giant stars in this sample would be a $20 \%$ increase in the number of currently known giant stars hosting a planet ( $\sim 50$ according to the tabulation by Jones et al. 2014a). As Jones et al. (2014a) mentions, there is a small separation cut-off for RG hosts. The current record-holder for smallest separation of an RV-detected planet RG host is HIP 67851b with $a=0.539$ AU (Jones et al. 2014b). The shortest period planet orbiting a giant star, Kepler 91b, is on a 6 day orbit (Lillo-Box et al. 2014). Most of the candidate planets orbiting giants lie between these two systems, with a few candidates closer than Kepler $91 \mathrm{~b}$.

Of the evolved stars in this sample, 23 are verified RC stars (Bovy et al. 2014). RC stars are metal-rich stars which have passed through the tip of the RGB and have contracted due to the ignition of core helium burning. It is expected that stars like the Sun may reach radii up to $1 \mathrm{AU}$ when they reach the tip of the RGB. Therefore, the presence of companion candidates orbiting RC stars at $a<1 \mathrm{AU}$ in this catalog (see Figure 9) is a surprising discovery. To investigate this further we compared the RC stars to RGs in this sample, but we consider only RGs with $[\mathrm{Fe} / \mathrm{H}]>-0.42([\mathrm{Fe} / \mathrm{H}]$ of the most metal-poor $\mathrm{RC}$ in this sample) and $2.4 \leqslant \log g \leqslant 3.3$ (the $\log g$ range of the $\mathrm{RC}$ stars) to eliminate possible effects from RV sensitivity issues. This also allows us to compare stars approximately half way up the giant branch to stars that have already passed through the tip of the RGB, and have achieved their largest extent. These 92 RG stars have 62 stellar-mass, 25 BD-mass, and 5 planetmass companion candidates compared to 18,5 and 0 for the 23 $\mathrm{RC}$ giants.

A cursory look at these numbers (and Figure 9) shows a lack of smaller companions for RC stars, as well as a companion candidates found at smaller separations for the $92 \mathrm{RG}$ stars when compared to RC stars (0.07 AU versus $0.2 \mathrm{AU}$ at the lowmass end). It is also interesting to note that no companion candidates have circular orbits among RC hosts (smallest $e=0.284$ ). This all points to the role of the tidal migration and destruction of companions, particularly planetary-mass companions. However, any tidally induced migration of companions will be much weaker than when the star was in the RGB phase. Therefore any companions with $a<1$ AU around an RC star likely would have to have survived inside the star's envelope during its RGB phase. Most of the RC hosts with $a<1 \mathrm{AU}$ candidate companions are likely post-common envelope systems, and thus may have experienced drag-induced migration to bring them to their current orbit. These systems certainly warrant deeper investigation. 


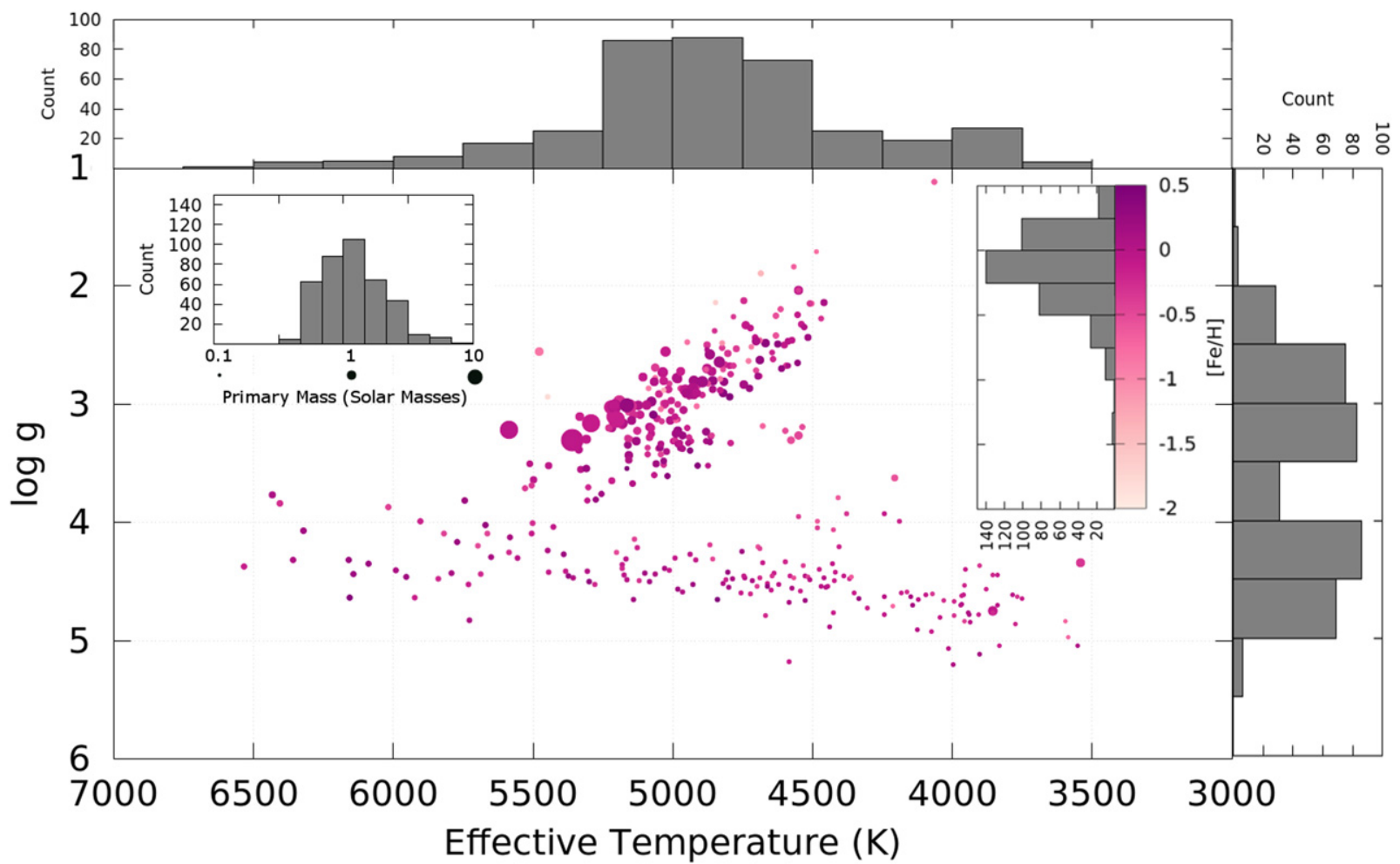

Figure 8. A spectroscopic HR diagram of the companion candidate hosting stars, with the host stars' $T_{\text {eff }}$ and $\log g$ as the abscissa and ordinate. The points are colorcoded by host star metallicity $([\mathrm{Fe} / \mathrm{H}])$, and point size indicates the primary mass in Solar masses. The stars along the bottom of the figure are the dwarf stars, and stars along the line connecting $\left(T_{\text {eff }}, \log g\right)=(5500 \mathrm{~K}, 3.5)$ and $(4000 \mathrm{~K}, 1)$ are the giants. Histograms of the effective temperature $\left(T_{\text {eff }}\right.$, top panel), surface gravity (log $g$, right panel), metallicity ([Fe/H], inset with color bar), and primary mass (inset with size legend) of the host stars in this gold sample are also shown.

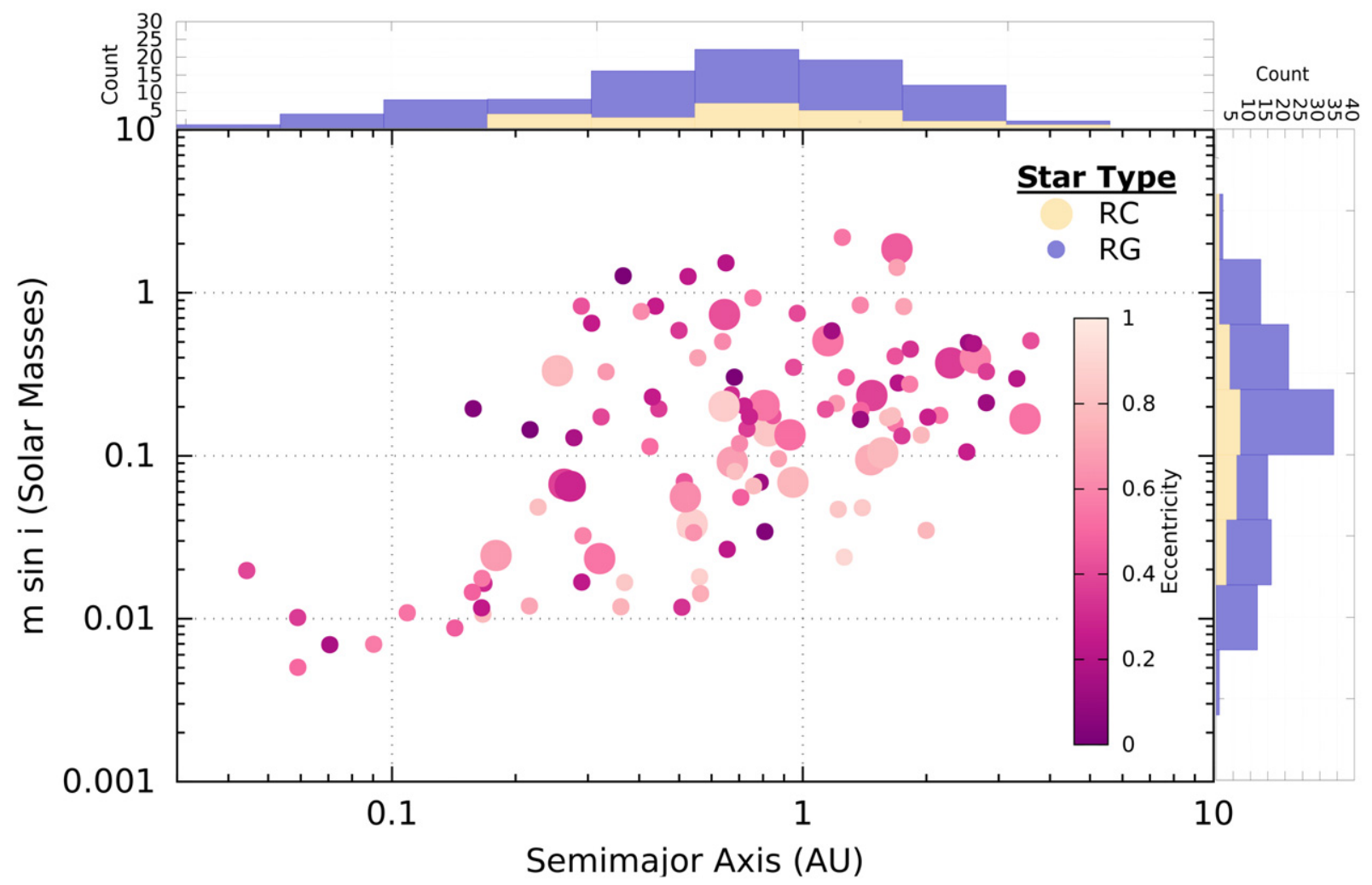

Figure 9. Orbital distribution of companion candidates to RC stars (large points) and RG stars with similar stellar parameters as this sample's RC stars (small points). Minimum orbital semimajor axis in AU is on the abscissa and minimum companion mass in $M_{\odot}$ is on the ordinate. Color again represents the orbital eccentricity of the companion. The panels above and to the right of main plot show the $m \sin i$ and semimajor axis distribution for the RG comparison sample (purple histogram) and RC (gold histogram) hosts. 

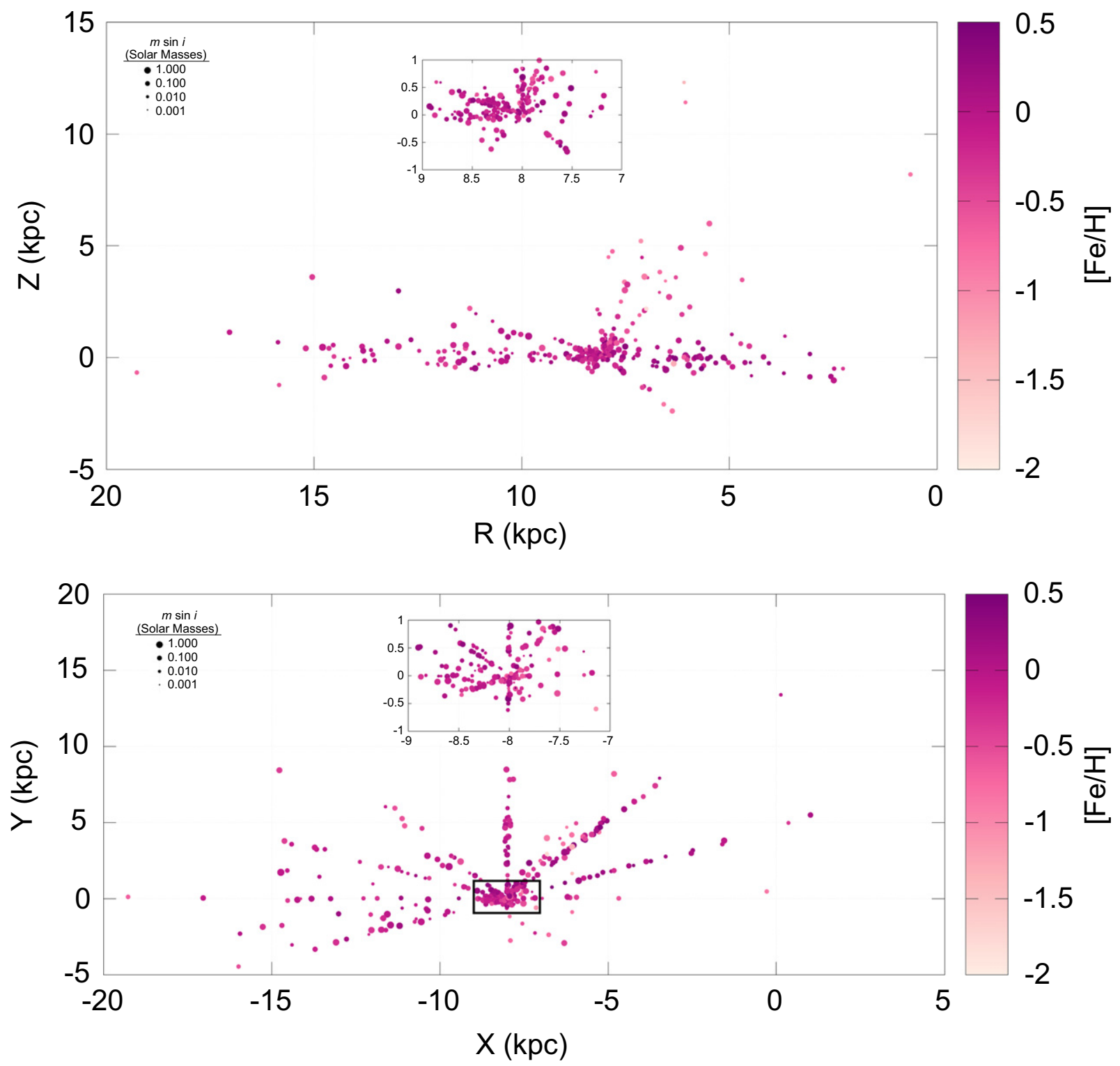

Figure 10. The Galactic distribution of companion candidate hosts in this catalog. Top Panel: distribution in Galactocentric $R$ and $Z$, where $R$ is the radial distance from the Galactic Center, and $Z$ is the height above the Galactic midplane. The color of the points indicates the metallicity ([Fe/H]) of host star, and the point size indicates $m \sin i$ of the companion candidate orbiting the star. The inset panel shows a detailed view of the solar neighborhood, which is indicated by the black box in the main plot $(7 \mathrm{kpc}<R<9 \mathrm{kpc},|Z|<1 \mathrm{kpc})$. Bottom Panel: distribution in Galactocentric $X$ and $Y$ rectilinear coordinates, where $(X, Y)=(0,0)$ and $(-8,0) \mathrm{kpc}$ are the locations of the Galactic Center and the Sun respectively, and $Y>0$ is in the direction of the Sun's orbit. The color and size of the points indicate the same data as they do in the top panel. Again, the inset panel shows a detailed view of the solar neighborhood, which is indicated by the black box in the main plot $(7 \mathrm{kpc}<X<9 \mathrm{kpc},|Y|<1 \mathrm{kpc})$.

\subsubsection{Metal-poor Companion Hosts}

According to the compilation of exoplanets.org (Han et al. 2014), of the confirmed planet-hosting stars with metallicity measurements, only 15 have $[\mathrm{Fe} / \mathrm{H}]<-0.5$. This sample has 41 stars with $[\mathrm{Fe} / \mathrm{H}]<-0.5$, and of these, two host candidate planetary-mass companions and 14 host candidate BD companions. The most metal-poor stars in this sample approach $[\mathrm{Fe} / \mathrm{H}]=-2$. While there are no candidate planets among the most metal-poor $([\mathrm{Fe} / \mathrm{H}]<-1)$ hosts (5 stars), there are two companions in the BD mass regime. The smaller fraction of the lowest-mass companions detected among the most metal-poor stars in this sample is not surprising as the RV uncertainties are higher for metal-poor stars, as described in Equation (1). Also, it is not too surprising to find metal-poor stars hosting binary companions, given the Carney et al. (2003) result. However, finding a population of metal-poor stars potentially hosting $\mathrm{BD}$ companions is surprising in the context of the core accretion model of companion formation, and may suggest an alternate formation mechanism for these companions.

\subsection{Galactic Distribution of Candidate Hosts}

Most surveys for stellar and substellar companions have focused on stars in the solar neighborhood, especially with the recent interest in M dwarf planet hosts. In contrast, only three of the sources in this catalog are within $100 \mathrm{pc}$ of the Sun, where the vast majority of known planets with distance measurements have been found. In a Galactic context, this 
sample is truly complementary to previous studies. The current most-distant known planet host is the microlensing source OGLE-2005-BLG-390 L at $6.59 \mathrm{kpc}$ (Beaulieu et al. 2006). The most-distant planetary-mass $\left(\langle m\rangle=7.26 M_{\text {Jup }}\right)$ candidate companion in this catalog orbits the slightly metal-poor $([\mathrm{Fe} /$ $\mathrm{H}]=-0.34), \mathrm{RG}(\log g=2.5)$ star $2 \mathrm{M} 05445028+2847562$, which lies at a comparable distance of $6.13 \mathrm{kpc}$.

Furthmore, this sample has 36 companion candidates farther than this distance. Of these, 12 are BD-mass companions around stars reaching to a distance of $15.7 \mathrm{kpc}$. Figure 10 demonstrates the Galactic reach of this catalog's companion candidate hosts. A large majority of this sample (327 stars) resides in the Galactic Thin Disk $(|Z|<1 \mathrm{kpc}$, but see note $\mathrm{f}$ in Table 1), but these disk stars reach from inner disk ( $R \sim 2 \mathrm{kpc})$ to the outer disk ( $R \sim 15 \mathrm{kpc})$. From this preliminary analysis, it is safe to say that companions of all types are ubiquitous across the thin disk. As we move from the thin disk to the halo, the proportion of higher-mass companions increases. This trend is likely due to the combination of the sensitivity bias that lowmass companions are less likely to be detected around more metal-poor stars (see Equation (1)), and the Planet-Metallicity correlation.

\section{FUTURE SURVEY DIRECTIONS}

The APOGEE-2 survey is a six-year extension of the APOGEE survey as a part of SDSS-IV. APOGEE-2 continues the survey of the northern hemisphere at APO, and implements a new component of the survey at the DuPont Telescope at Las Campanas Observatory (LCO) to cover the southern hemisphere. A dedicated search for substellar companions was approved as a goal science program in APOGEE-2. By the end of APOGEE-2, in 2020, we will have acquired $\geqslant 24$ epochs of RV measurements of 1074 red giant stars across 5 fields, including fields containing the star cluster NGC 188 as well as a COROT (Bordé et al. 2003) field. These fields were selected to search for companions because of previous observations from APOGEE-1. Many of these targets will accrue up to a 9 year temporal baseline of observations.

In addition to the planned dedicated fields, we expect many additional APOGEE-2 fields will have candidates discovered serendipitously, as they were with this work. In APOGEE-1, $\sim 10 \%$ of the targeted stars had $\geqslant 8$ visits, and of those, $\sim 2.6 \%$, or a cumulative $\sim 0.26 \%$ of all survey stars, were selected as having companion candidates. APOGEE-2 will bring the cumulative total number of stars observed by the APOGEE instrument to $\sim 500,000$ stars. Therefore, assuming a similar detection rate and visit distribution, as well as the "gold sample" selection criterion used here, we expect to detect a cumulative total of at least $\sim 1300$ companion candidates by the end of APOGEE-2.

Several technical improvements to the APOGEE pipelines planned for SDSS-IV will improve RV precision, and as a result, improve our ability to measure the orbital and physical parameters of systems observed in APOGEE-2 as well as the current sample of candidates. One upgrade to ASPCAP of particular importance to our efforts is the implementation of stellar rotational velocity determination to ensure more reliable parameters for dwarf stars and rapidly rotating giants. In addition, acquiring rotational velocities will allow estimates of the ages of the dwarf star hosts in future catalogs through the age-rotation correlation. We will also reap the rewards of a fully vetted and improved distance catalog.
We have a significant ongoing observational program to individually investigate the best planetary mass and BD systems in this catalog that includes high-resolution spectroscopy, diffraction-limited imaging, and photometric variability monitoring. The results of these efforts will be included in future versions of this catalog.

\section{CONCLUSIONS}

Through analysis of multiple epochs of APOGEE spectroscopic data, we have identified 382 stars that have strong candidates for stellar and substellar companions, of which 376 had no previous reports of small separation companions. From an initial analysis of this sample we have found:

1. Two distinct regimes of companions in $m \sin i-a$ space exist that are likely the result of distinct formation paths for stellar-mass and planetary-mass companions, with the gap between the two regimes being a manifestation of the BD desert. However, we find a smaller and "wetter" BD desert with the BD desert only manifesting itself for orbital separations of $a<0.1-0.2 \mathrm{AU}$ in this sample of candidate companions, much smaller than the $5 \mathrm{AU}$ proposed in previous studies. We proposed a few potential explanations of this result: (a) lower mass MS candidate hosts host a higher relative number of BD-mass candidates than their higher-mass MS counterparts, lending evidence to the Duchêne \& Kraus (2013) interpretation that the $\mathrm{BD}$ desert may be a special case of a more general dearth of extreme mass ratio binary systems. (b) A majority of the candidate BD companions in this catalog orbit evolved F dwarfs, supplying further evidence to the " $F$ dwarf oasis" hypothesis proposed for small separation BD companions by Guillot et al. (2014). (c) The possibility of planetary-mass candidates orbiting within $\sim 1$ AU initiating Roche lobe overflow of their hosts as it ascends the giant branch, allowing planetarymass companions to grow to BD mass.

2. A significant number of small-separation eccentric systems which may be evidence for ongoing tidal migration among the giant hosts and the eccentricity-pumping mechanism proposed by Tsang et al. (2014) for the dwarf hosts.

3. A set high mass ratio candidate systems $(\langle q\rangle>0.5)$, of which 28 show indications of containing a stellar remnant, including two neutron stars, and eight potential He-core white dwarfs.

4. 225 candidate companions orbiting evolved (RC, RG, and SG) stars. This includes nine new planetary-mass candidate companions around giant stars, which, if confirmed, would be a $>20 \%$ increase from the previously known number given by Jones et al. (2014a), as well as 3 planetary-mass candidates orbiting subgiant stars. Among the RC stars, 15 host companion candidates orbiting within $1 \mathrm{AU}$, the maximum expected extent of a RGB star evolved from a Sun-like star, indicating these systems are likely post-common envelope systems.

5. A population of 41 metal-poor $([\mathrm{Fe} / \mathrm{H}]<-0.5)$ candidate companion hosting stars, of which 2 host planetarymass candidates, and 14 host BD candidates. These systems challenge the planet-metallicity correlation, and thus the core accretion paradigm of companion formation. It is possible the formation pathway for these 
companions may closer mimic that of binary systems or a gravitation instability scenario.

6. To first order, companions of all kinds are prevalent throughout the disk $(2 \mathrm{kpc}<R<15 \mathrm{kpc},-2 \mathrm{kpc}<Z$ $<2 \mathrm{kpc}$ ), with planetary-mass companions found out to distances of $\sim 6 \mathrm{kpc}$, and $\mathrm{BD}$-mass companions to distances of $\sim 16 \mathrm{kpc}$.

A campaign is underway to confirm and further characterize the nature of the candidate companion systems reported here. This effort will be augmented with SDSS-IV APOGEE-2 observations. Between APOGEE-1 targets obtaining additional visits and new APOGEE-2 targets obtaining a large number of visits, we expect APOGEE's sample of candidate companions to at least triple by the end of SDSS-IV.

The research described in this paper makes use of Filtergraph, an online data visualization tool developed at Vanderbilt University through the Vanderbilt Initiative in Dataintensive Astrophysics (VIDA). Particular thanks to Dan Burger who quickly answered questions and solved problems as they occurred.

This research has made use of the NASA Exoplanet Archive, which is operated by the California Institute of Technology, under contract with the National Aeronautics and Space Administration under the Exoplanet Exploration Program.

This research has made use of the Exoplanet Orbit Database and the Exoplanet Data Explorer at exoplanets.org.

Funding for SDSS-III has been provided by the Alfred P. Sloan Foundation, the Participating Institutions, the National Science Foundation, and the U.S. Department of Energy Office of Science. The SDSS-III web site is http://www.sdss3.org/.

SDSS-III is managed by the Astrophysical Research Consortium for the Participating Institutions of the SDSS-III Collaboration including the University of Arizona, the Brazilian Participation Group, Brookhaven National Laboratory, Carnegie Mellon University, University of Florida, the French Participation Group, the German Participation Group, Harvard University, the Instituto de Astrofisica de Canarias, the Michigan State/Notre Dame/JINA Participation Group, Johns Hopkins University, Lawrence Berkeley National Laboratory, Max Planck Institute for Astrophysics, Max Planck Institute for Extraterrestrial Physics, New Mexico State University, New York University, Ohio State University, Pennsylvania State University, University of Portsmouth, Princeton University, the Spanish Participation Group, University of Tokyo, University of Utah, Vanderbilt University, University of Virginia, University of Washington, and Yale University.

D. L. N. was supported by a McLaughlin Fellowship at the University of Michigan. J. K. C. was supported by an appointment to the NASA Postdoctoral Program at the Goddard Space Flight Center, administered by Universities Space Research Association through a contract with NASA. Szabolcs Meszaros has been supported by the János Bolyai Research Scholarship of the Hungarian Academy of Sciences. C. A. P., D. A. G. H., and O. Z. acknowledge support provided by the Spanish Ministry of Economy and Competitiveness (MINECO) under grants AYA2014-56359-P, RYC-201314182, and AYA-2014-58082-P.

We would like to extend our gratitude to Phil Arras (Virginia) and Kaitlin Kratter (Steward Observatory) for their most useful theoretical insights on our results.

Facility: Sloan.

\section{APPENDIX A VERIFICATION AND PERFORMANCE}

As with all survey reduction pipelines, the goal is to balance speed and accuracy. Our code is reasonably fast, with a typical star taking 30-60 s for a complete fit to be performed, as described above. Below, we describe the efforts to verify the accuracy of the apOrbit pipeline.

\section{A.1. Simulated Systems}

RV curves were generated for a suite of simulated systems to verify the output of the apOrbit pipeline. The simulations mimic the observations of candidate planet hosting stars by the APOGEE survey (see Section 2.8 of Majewski et al. 2015), and can be used to investigate the types of systems that can be identified and characterized in the APOGEE-1 survey.

\section{A.1.1. Generation of Simulated Systems}

We simulated 9000 planetary systems with random characteristics. The masses of the primary stars were drawn from the distribution of estimated masses for the actual candidate substellar hosts in the APOGEE data. The companion masses and periods were drawn from the distributions specified by Tabachnik \& Tremaine (2002), using a mass range of 1-100 $M_{\text {Jup }}$ and periods from 0.1 to 2000 days. Eccentricities were drawn from a uniform distribution with a maximum of $e=0.934$, which corresponds to the eccentricity of $\mathrm{HD}$ 80606 b, the largest eccentricity in the exoplanets.org. database. Companions with $P<5$ days were assumed to have circular orbits. The radii of the APOGEE candidate host stars were also estimated, and planets with orbital separations less than $5 R_{\star}$ were considered unphysical because the tidal decay of planetary orbits becomes relevant at such small separations. The longitude of periastron and the orbital phase of periastron passage relative to a reference date were drawn from uniform distributions.

With the orbital characteristics of the simulated companions defined, we simply used the helio_rv code in the IDL astronomy users library ${ }^{27}$ to calculate the measured heliocentric $\mathrm{RV}$ for each system on a set of observation dates. The observation dates for each system were designed to mimic the way the survey proceeded. The observations for each star were spread randomly over a 3.2 year time period assuming the telescope was on-sky for 15 days followed by 14 days off sky since APOGEE observed primarily during bright time. The simulated measured RVs consisted of the actual motion of the star at the time of observation plus two sources of noise, drawn from Gaussian distributions. The first is simply measurement noise, which nominally has $\sigma_{v}=100 \mathrm{~m} \mathrm{~s}^{-1}$ but is increased to $\sigma_{v}=130 \mathrm{~m} \mathrm{~s}^{-1}$ for $20 \%$ of the visits to simulate poor observing conditions. The second noise source is intrinsic stellar atmospheric RV jitter, with an amplitude drawn from the distribution in Frink et al. (2001).

A second data set of 9000 simulated system were generated with much of the same parameters as the first, except that it had mass ratios approaching one, all orbital parameters were drawn from a uniform distribution, and it was much sparser in the lower-mass companion regime. We combined these two data sets to obtain complete coverage of parameter space. From the combined data set, we generated RV curves with 9, 12, 16, and

27 http://idlastro.gsfc.nasa.gov 


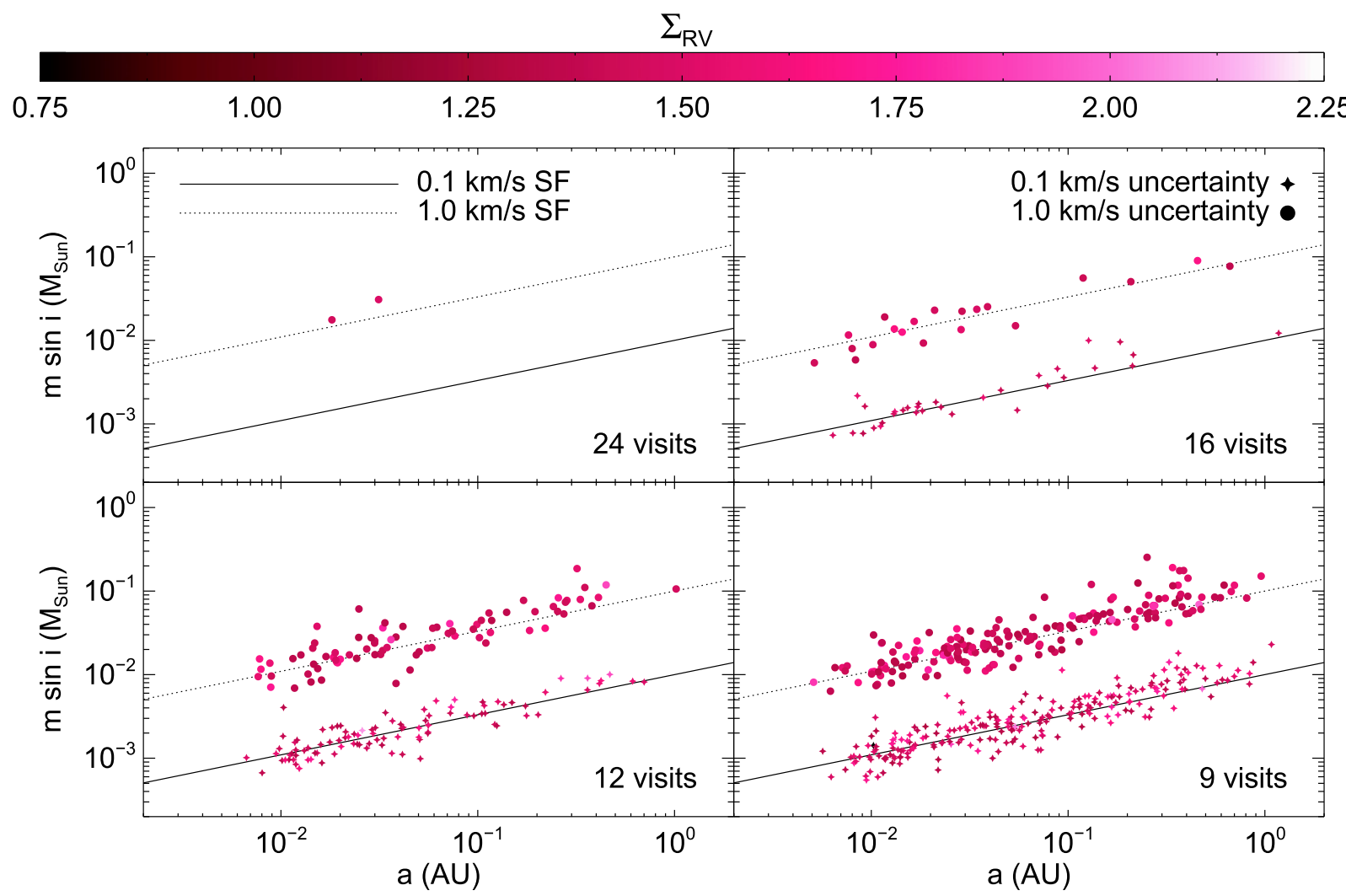

Figure 11. Distribution of false positive companions in recovered $m \sin i-a$ space, with color representing the $\Sigma_{\mathrm{RV}}$ statistic (see Equation (2)). Each set of points (unique panel and shape) is drawn from a sample of 18,000 simulated systems with 9 (bottom right panel), 12 (bottom left panel), 16 (top right panel), and 24 (top left panel) simulated RV measurements based on stellar jitter and random measurement error. Symbol shape indicates the uncertainty level used for the simulation, with circles indicating $1 \mathrm{~km} \mathrm{~s}^{-1}$ and four-point stars indicating $0.1 \mathrm{~km} \mathrm{~s}^{-1}$ uncertainties. The solid and dotted lines show the approximate sensitivity function (SF; see Equation (32)) for 0.1 and $1 \mathrm{~km} \mathrm{~s}^{-1} \mathrm{RV}$ uncertainties. False positive signals such as these are removed from the sample via the velocity cut described in Section 3.1.2.

24 visits selected from the 24-visit parent sample with the $100 \mathrm{~m} \mathrm{~s}^{-1} \mathrm{RV}$ uncertainty level, as well as a set where the base uncertainty level is inflated to $1 \mathrm{~km} \mathrm{~s}^{-1}$ to emulate $\mathrm{RV}$ measurements from the metal-poor host stars in this sample.

\section{A.1.2. Determination of Quality Criteria and False Positive Analysis}

The full test suite of simulated systems was run through the apOrbit pipeline each time an update to the fitting algorithms was implemented. Many of these updates were inspired by the simulated systems for which the pipeline failed to reproduce the correct orbit in the previous run. In addition, many of the criteria used to select the RV variable and gold candidate sample, described in Section 4, were inspired by these results. Notable failures in previous runs that led to new selection criteria for candidate companions included:

1. Long-Period Systems: the longest-period simulated systems demonstrated the largest scatter in their results. This inspired the use of the phase uniformity index (see Section 3.3.4), as well as a procedure to reject any solutions for which the period was longer than twice the baseline (Section 4.2).

2. Highly Eccentric Systems: the code had the most difficulty reproducing the orbital parameters of systems with high eccentricity $(e>0.9)$. However, these systems are extremely rare, so this is not a major issue. Nevertheless, this result still led to the decision to reject all orbital solutions with $e>0.934$ (Section 3.3.4), which is the planetary system with the largest known eccentricity anyway. Even with this cut, however, the more eccentric the system, the more trouble the code had in recovering the correct orbital parameters. In particular, systems with low numbers of visits had the most issues. This result inspired the use of the velocity uniformity index in the fitting code (Section 3.3.4), and it led to the decision to implement more stringent significance cuts for eccentric systems (Section 4.1).

3. One-day Aliased Systems: early tests of the code on simulated systems revealed a tendency for solutions to cluster around integer fractions of one day, despite the initial period selection avoidance of such periods. This inspired the decision to reject any periods within $5 \%$ of $1 / 3,1 / 2,1,2$, or 3 days (Section 3.3.4).

These simulations were also used to understand how RV noise from the star or measurement error can potentially lead to a false positive candidate companion. To accomplish this we ran the simulated systems through the apOrbit pipeline following the procedures laid out in Section 3.3 using just the RV signals from the star's atmospheric jitter and random measurement errors. We ran the simulations using four different numbers of visits $(9,12$, 16 , and 24) and two uncertainty levels $\left(0.1\right.$ and $\left.1 \mathrm{~km} \mathrm{~s}^{-1}\right)$, and selected candidates using the criteria described in Section 4. The results of the false positive tests are summarized in Figure 11. For systems with 24 visits, out of 18,000 simulated systems only two systems at the $1 \mathrm{~km} \mathrm{~s}^{-1}$ uncertainty level registered as false positives. The raw number of false positives increased dramatically from 24 to 16 visits, and the higher uncertainties 


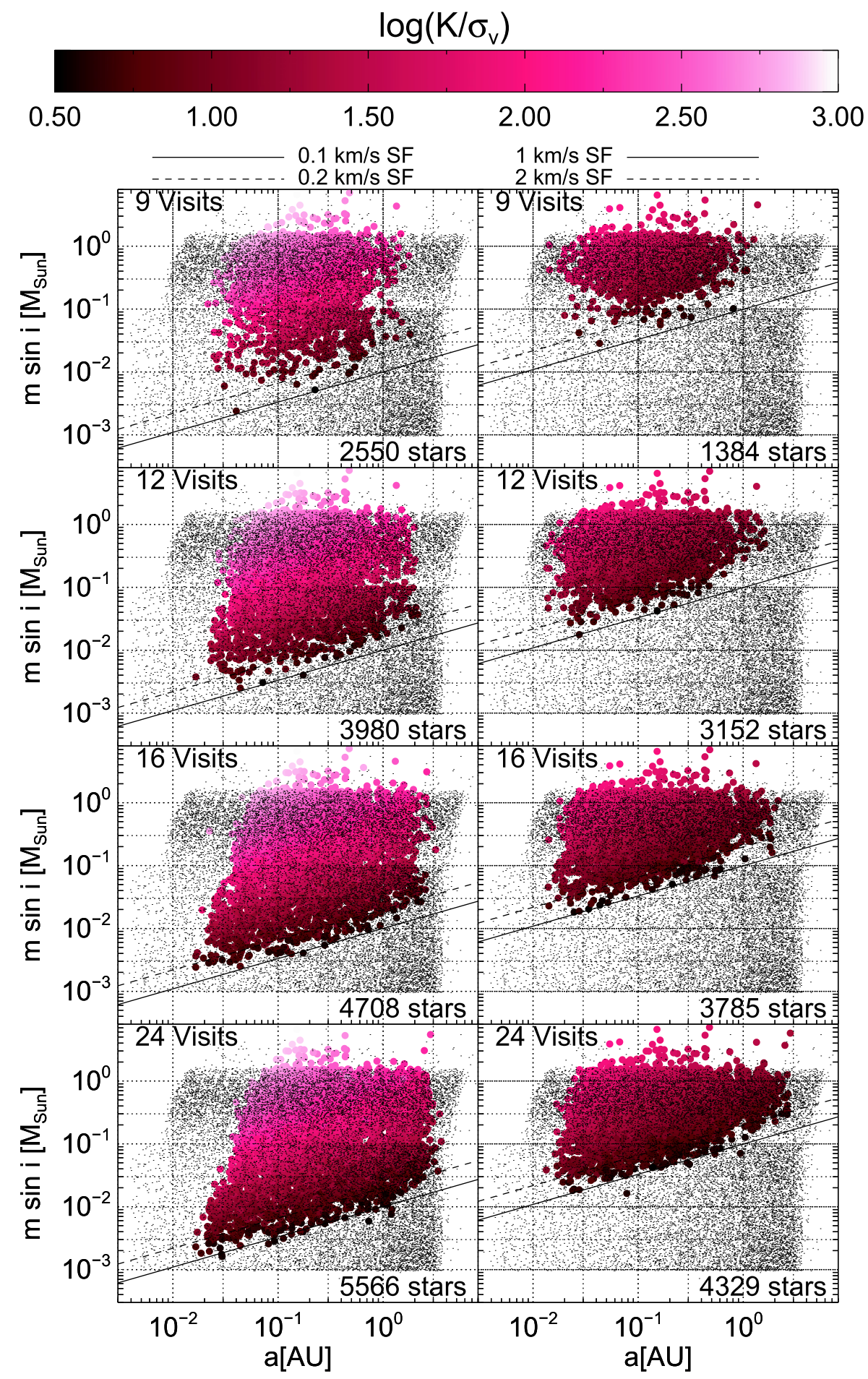

Figure 12. The gray dots are the locations of the 18,000 simulated systems in actual $m \sin i$-a space. The colored circles indicate the correctly recovered systems that were selected as candidates using the same metric as the "gold sample," color-coded by recovered $\log (K / \sigma)$, with the number of systems correctly recovered indicated in the bottom right corner of each panel. The black solid and dashed lines mark the sensitivity function (SF; see Equation (32)) for the baseline and twice the baseline $\mathrm{RV}$ uncertainties of $0.1 \mathrm{~km} \mathrm{~s}^{-1}$ (left column) and $1.0 \mathrm{~km} \mathrm{~s}^{-1}$ (right column) used by the simulations. The simulations presented here emulate stars with, from top row to bottom row, $9,12,16$, and 24 visits.

lead to a higher rate of false positives. Most false positives clustered around the sensitivity limit corresponding to the RV measurements from which they were derived (see Equation (32) below), and are generally assigned eccentric orbits $(e>0.5)$. This information, along with visual inspection of these fits, led to a pre-cut based on the velocity variations of the star which was based on the value of the statistic described in Section 3.1.2 for these systems.

\section{A.1.3. Sensitivity Limit, Parameter Accuracy, and Recovery Rate}

In the results presented here, we ran the 18,000 simulated systems through the apOrbit pipeline as described in 

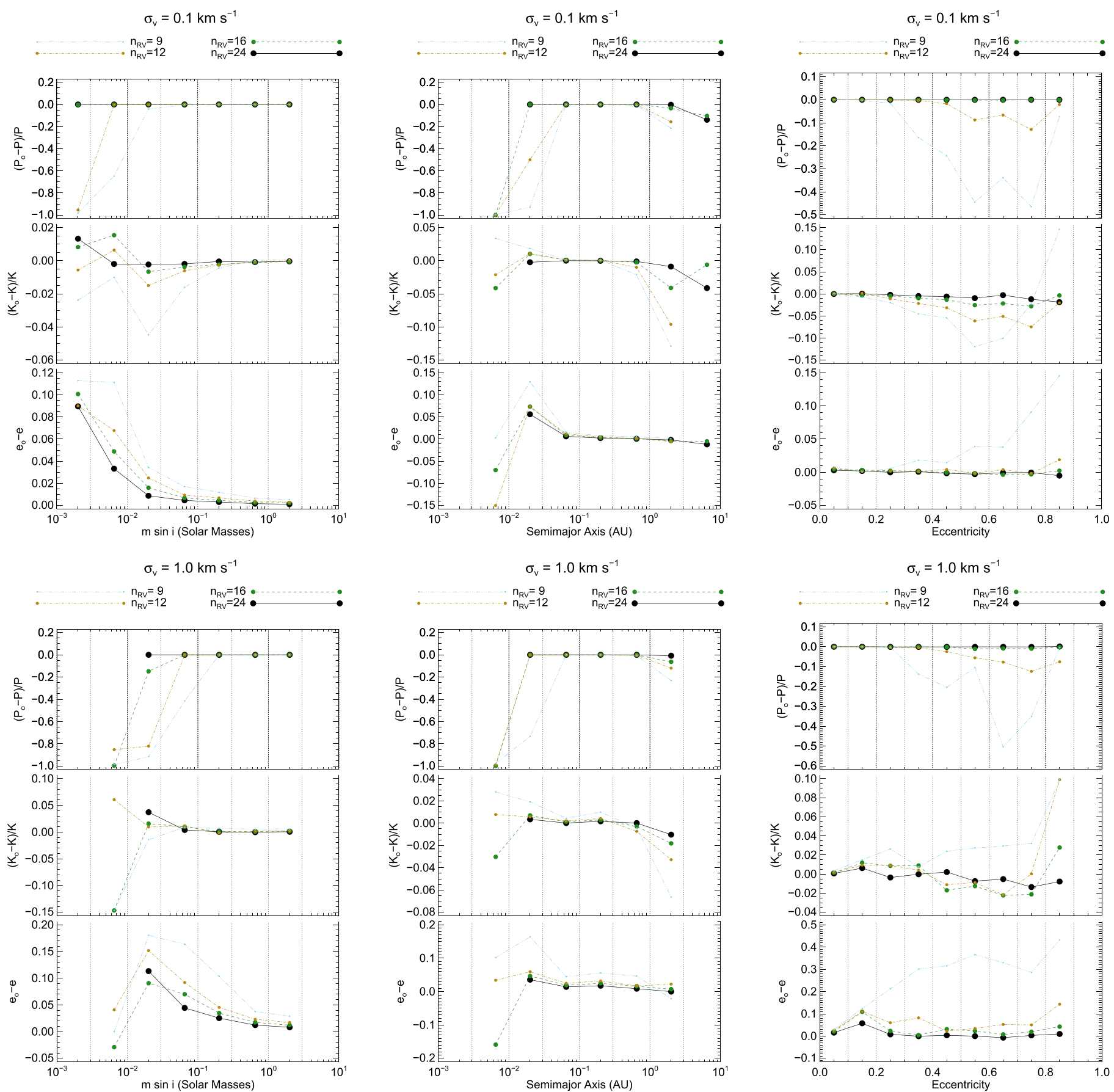

Figure 13. The recovered simulated systems binned by their recovered companion mass ( $m \sin i$; left column), semimajor axis ( $a$; center column), and eccentricity ( $e$; right column). In each plot, the ordinates are the fractional error in period (top panel), fractional error in semiamplitude (middle panel), and error in eccentricity (bottom panel), where $X_{o}$ indicates the recovered value of parameter with true value $X$. The top row of plots present the results using a base RV uncertainty of $\sigma_{v}=0.1$ $\mathrm{km} \mathrm{s}^{-1}$, and the bottom row shows $\sigma_{v}=1 \mathrm{~km} \mathrm{~s}^{-1}$. Cyan, tan, green, and black points (dash triple-dotted, dash dotted, dashed, and solid lines) are from simulations with $9,12,16$, and 24 visits, respectively. The vertical dotted lines mark the bins used, and for any bin with $<3$ stars, the point is excluded.

Section 3.3.2 for the four visit levels $\left(n_{\mathrm{RV}}=9,12,16,24\right)$ and two uncertainty levels $\left(\sigma_{v}=0.1,1 \mathrm{~km} \mathrm{~s}^{-1}\right)$, and selected companion candidates in the same manner as the gold sample, as described in Sections 3.1.2, 3.3.4, and 4. The systems correctly recovered $(P$ and $K$ recovered within $10 \%$, and $e$ recovered within 0.1 ) are shown in Figure 12. Systems with large $K$ but small errors can lead to larger values of $\chi^{2}$ for a fit that still produces the correct orbital parameters. Unfortunately, removing the $\chi_{\text {mod }}^{2}$ constraint allows many systems with incorrect solutions to pass, so we err on the side of caution and keep it in place. Due to limits of the period search and the other constraints on the period, $a$, and $\chi_{\text {mod }}^{2}$ of the fit described in Section 4.2, we expect to be able to recover orbits for companions having $0.01 \mathrm{AU} \lesssim a \lesssim 3 \mathrm{AU}$, depending on the baseline, number of visits, and the RV uncertainty level (see Figure 12). By fitting a trendline to the simulated systems with $2.8 \leqslant K / \tilde{\sigma}_{v} \leqslant 3$ for various values of $\sigma_{v}$, we also find that the lower limit on detectability, which we 

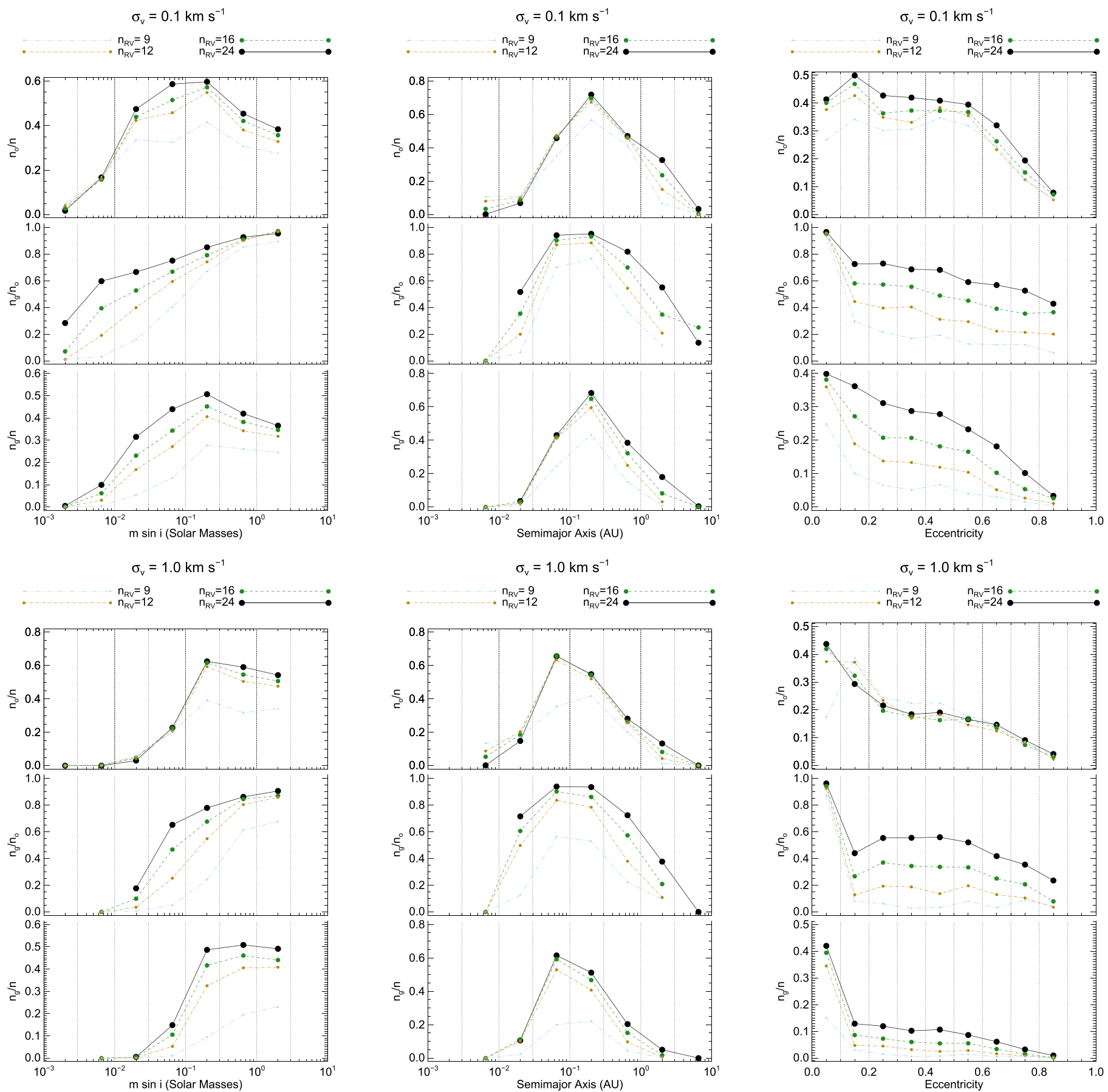

Figure 14. The same as Figure 13, except on the ordinate in each plot, the top panel shows the fraction of systems recovered in the bin, $n_{o}$, compared to the total number of simulated systems in the bin, $n$, the middle panel shows the fraction of systems recovered with correct orbital parameters $(P$ and $K$ within $10 \%$ and $e$ within $0.1), n_{g}$ compared to $n_{o}$, and the bottom panel shows $n_{g} / n$. Here a bin is excluded if the denominator of the ordinate is $<2$.

will refer to as the sensitivity function (SF), of $m \sin i$ can be written as:

$$
\log (m \sin i)=0.48 \log (a)-C,
$$

where the constant offset, $C$, depends on the sensitivity level (which we interpret as the median RV uncertainty, $\tilde{\sigma}_{v}$ ):

$$
C / \log \left(M_{\odot}\right)=2.0-0.3 \log _{2}\left(\tilde{\sigma}_{v} / 100 \mathrm{~m} \mathrm{~s}^{-1}\right) .
$$

We then compared each observed/recovered orbital parameter, $X_{o}$, with the actual parameters for the system, $X$, to determine how accurately the parameters are recovered as a function of parameter space. These results are summarized in Figure 13. For a large portion of the parameter space, the selected candidates reproduce the correct orbital parameters quite well. The systems that give the most trouble appear to be the low-mass companions, companions at large separations, and companions with highly eccentric orbits. Unsurprisingly, parameter recovery is overall worse for stars with fewer visits and higher RV uncertainties, but the drop in performance was not as dramatic between the 24 visit and 16 visit simulations as 
Table 2

KOIs and Kepler EBs Selected as Gold Sample Companion Candidates

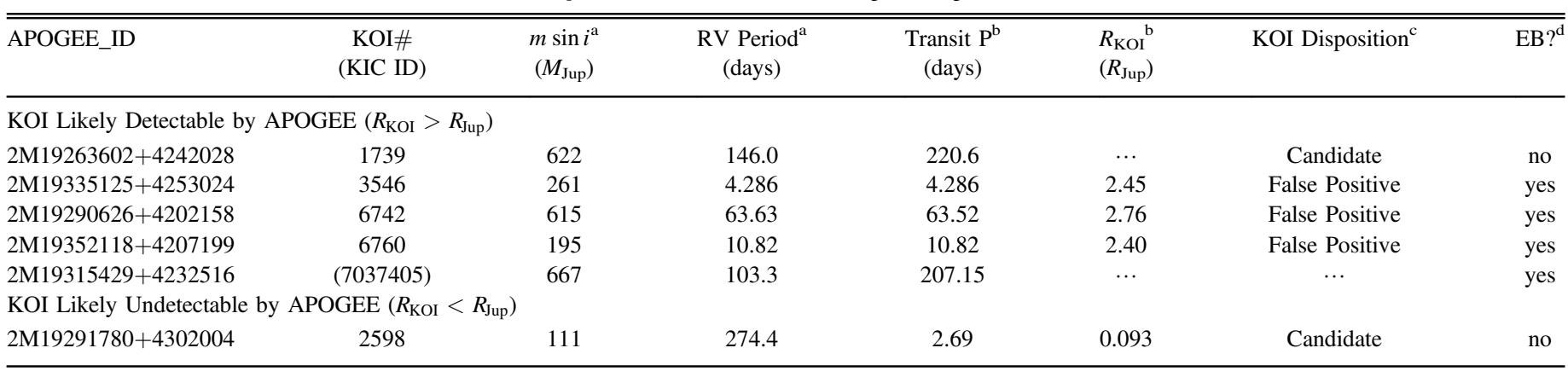

Notes.

a As determined by APOGEE RV data (this work).

b As determined by Kepler transit data (Mullally et al. 2015).

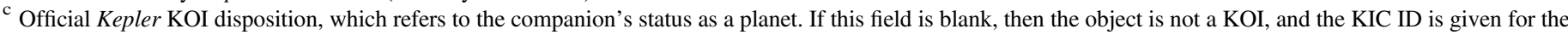

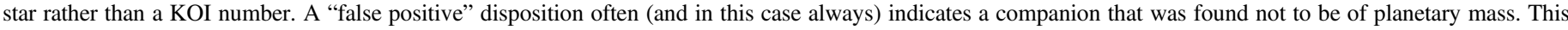

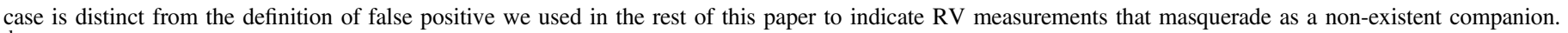

${ }^{\mathrm{d}}$ Is the star in the Kepler Eclipsing Binary catalog (Slawson et al. 2011; LaCourse et al. 2015)?

Table 3

Comparison of Recovered Parameters of Known Exoplanet System HD $114762 \mathrm{~B}$

\begin{tabular}{lcc}
\hline \hline Parameter & APOGEE Value & Literature $^{\text {a }}$ Value \\
\hline Host Stellar Parameters & & \\
$T_{\text {eff }}$ & $5466 \mathrm{~K}$ & $5673 \mathrm{~K}$ \\
$\log g$ & 4.196 & 4.135 \\
{$[\mathrm{Fe} / \mathrm{H}]$} & -0.832 & -0.774 \\
Distance & $36.5 \mathrm{pc}$ & $38.7 \mathrm{pc}$ \\
$M_{\star}$ & $0.86 M_{\odot}$ & $0.83 M_{\odot}$ \\
$R_{\star}$ & $1.20 R_{\odot}$ & $1.24 R_{\odot}$ \\
Orbital Parameters & & $83.92 \mathrm{days}$ \\
$P$ & $85.58 \mathrm{days}$ & $612.5 \mathrm{~m} \mathrm{~s}$ \\
$K$ & $925.5 \mathrm{~m} \mathrm{~s}$ & 0.335 \\
$e$ & 0.594 & $10.98 M_{\mathrm{Jup}}$ \\
$m \sin i$ & $14.72 M_{\mathrm{Jup}}$ & $0.353 \mathrm{AU}$ \\
$a$ & $0.361 \mathrm{AU}$ & \\
\hline
\end{tabular}

Note.

${ }^{\mathrm{a}}$ Kane et al. (2011).

it was between the 16 visit to 9 visit simulations. However, from these results we can still conclude that in almost all regimes, the recovered orbital parameters are at least characteristic of the true values for the system.

Finally, we construct the recovery rates across the parameter space covered by this catalog. These are summarized in Figure 14. Unsurprisingly, recovery rate drops as $n_{R V}$ decreases, and higher RV uncertainties lead to lower recovery rates in general.

\section{A.2. Comparisons with Systems with Known Companions}

In addition to comparing to the known parameters of simulated RV signals, we also compared our results to the transit periods of 5 Kepler object of interest (KOI) hosts and one non-KOI eclipsing binary (EB) observed by APOGEE (Fleming et al. 2015) that also meet the gold sample selection criteria described in Section 4. This comparison is presented in Table 2. We use the radius of the KOI as determined by Kepler transit data to split the sample. We assume a KOI with $R_{\mathrm{KOI}}=R_{\mathrm{Jup}}$ will have $m \sin i \approx M_{\mathrm{Jup}}$, and therefore any $\mathrm{KOI}$ with a radius less than this will likely be undetectable by APOGEE.

For three of the five APOGEE-detectable KOIs and EBs in the gold sample, the transit period is reproduced almost exactly, and for the remaining two, it appears that the aporbit code simply selected the wrong harmonic for the period. For example, with KOI-1739, if we assume that the 146.0 day period found by APOGEE is the first harmonic of a fundamental period of 73 days, then the first harmonic period would be 219 days, which is much closer to the transit period. Most of the APOGEE-detectable KOIs have been designated as "False Positives" by the Kepler team, meaning that the companion detected is not a planet, but rather a binary star companion. KOI-1739 is still designated as a candidate, but from APOGEE's RV data, we can conclude that this KOI should have a "false positive" disposition, as its companion is almost certainly not of planetary mass according to the analysis presented here. For the APOGEE-undetectable KOI, the APOGEE results from KOI-2598 may be indicative of longerperiod companion previously undetected by transit. Further investigation of these this system is certainly warranted.

Furthermore, APOGEE recovered the known planet HD 114762b (2M13121982+1731016), with which we compare orbit parameters and host stellar parameters derived and adopted by the aporbit pipeline to literature values in Table 3. APOGEE's recovered stellar parameters, as well as the recovered period and orbital semimajor axis are in good agreement with the results from Kane et al. (2011), but APOGEE overestimates the eccentricity of the system, and thus the values of $K$ and $m \sin i$. This is in agreement with our findings in Appendix B.1. However, this star was selected for use as a telluric standard, and thus is not included in our gold sample. This result may lead us to reconsider excluding stars selected as telluric standards in future versions of this catalog, especially considering the upgrades described in Section 6 which will lead to improved stellar parameters and RV determinations for dwarfs.

\section{APPENDIX B CATALOG INFORMATION}

For each star in the 382-star gold sample, the following data are available: 
Table 4

Data Model for the 1st Extension of the goldorbit-dr12.fits File

\begin{tabular}{|c|c|c|c|}
\hline Field Name & Data Type & Units & Description \\
\hline APOGEE_ID & $\operatorname{char}[18]$ & $\ldots$ & TMASS-STYLE object name \\
\hline LOCATION_ID & int16 & $\cdots$ & APOGEE field location ID number \\
\hline FIELD & $\operatorname{char}[16]$ & $\cdots$ & APOGEE field name \\
\hline NVISITS & int16 & $\cdots$ & Number of RV measurements used in the fit \\
\hline SNR & float32 & $\cdots$ & median $\mathrm{S} / \mathrm{N}$ per pixel in combined frame (at apStar sampling) \\
\hline $\mathrm{J}$ & float32 & mag & 2MASS J mag \\
\hline J_ERR & float32 & mag & uncertainty in 2MASS J mag \\
\hline$\overline{\mathrm{H}}$ & float32 & mag & 2MASS H mag \\
\hline H_ERR & float32 & mag & uncertainty in 2MASS $\mathrm{H}$ mag \\
\hline $\mathrm{K}$ & float32 & mag & 2MASS Ks mag \\
\hline K_ERR & float32 & mag & uncertainty in 2 MASS Ks mag \\
\hline $\mathrm{AK}$ & float32 & mag & $K$-band extinction adopted \\
\hline AK_SRC & $\operatorname{char}[17]$ & $\ldots$ & Method used to get targeting extinction \\
\hline RA & float64 & degrees & Right ascension (J2000) \\
\hline DEC & float64 & degrees & Declination (J2000) \\
\hline GLON & float64 & degrees & Galactic longitude \\
\hline GLAT & float64 & degrees & Galactic latitude \\
\hline PMRA & float32 & $\operatorname{mas} \mathrm{yr}^{-1}$ & One proper motion measurement \\
\hline PMDEC & float32 & mas $\mathrm{yr}^{-1}$ & One proper motion measurement \\
\hline PM_SRC & $\operatorname{char}[20]$ & $\cdots$ & Catalog used for PM \\
\hline EXTRATARG & int32 & $\ldots$ & bitmask that identifies main survey targets and other classes ${ }^{\mathrm{a}}$ \\
\hline APOGEE_TARGET1 & int32 & $\cdots$ & bitwise OR of first APOGEE target flag of all visits ${ }^{\mathrm{a}}$ \\
\hline APOGEE_TARGET2 & int32 & $\cdots$ & bitwise OR of second APOGEE target flag of all visits ${ }^{\mathrm{a}}$ \\
\hline TARGFLĀGS & $\operatorname{char}[116]$ & $\ldots$ & target flags in English \\
\hline STARFLAG & int 32 & $\cdots$ & Flag for star condition taken from bitwise OR of individual visits ${ }^{\mathrm{a}}$ \\
\hline STARFLAGS & char[129] & $\cdots$ & STARFLAG in English \\
\hline ASPCAPFLAG & int32 & $\cdots$ & Flag for ASPCAP analysis ${ }^{a}$ \\
\hline ASPCAPFLAGS & $\operatorname{char}[114]$ & $\ldots$ & ASPCAPFLAG in English \\
\hline TEFF & float32 & $\mathrm{K}$ & Adopted $T_{\text {eff }}$ for the primary star \\
\hline TEFF_ERR & float32 & $\mathrm{K}$ & Adopted $T_{\text {eff }}$ uncertainty \\
\hline LOGG & float32 & $\log (\mathrm{cgs})$ & Adopted $\log g$ for the primary star \\
\hline LOGG_ERR & float32 & $\log (\mathrm{cgs})$ & Adopted $\log g$ uncertainty \\
\hline FE_H & float32 & dex & Adopted $[\mathrm{Fe} / \mathrm{H}]$ for the primary star \\
\hline FE_H_ERR & float 32 & dex & Adopted $[\mathrm{Fe} / \mathrm{H}]$ uncertainty \\
\hline SPARAMTYPE & int16 & $\cdots$ & Source of the stellar parameters adopted ${ }^{\mathrm{b}}$ \\
\hline STARTYPE & $\operatorname{char}[3]$ & $\cdots$ & Classification applied to host star (see Section 3.2.1) \\
\hline MSTAR & float 32 & $M_{\odot}$ & Mass of the primary based on the available stellar parameters \\
\hline MSTAR_ERR & float32 & $M_{\odot}$ & Uncertainty of the primary mass \\
\hline RSTAR & float32 & $\mathrm{AU}$ & Radius of the primary based on the available stellar parameters \\
\hline RSTAR_ERR & float32 & $\mathrm{AU}$ & Uncertainty of the primary radius \\
\hline DIST & float32 & $\mathrm{pc}$ & Adopted distance of the primary star \\
\hline DIST_ERR & float32 & $\mathrm{pc}$ & Uncertainty of the distance \\
\hline MSTAR_SRC & int16 & $\cdots$ & Source/method of mass/radius/distance estimation ${ }^{c}$ \\
\hline VJITTER & float 32 & $\mathrm{~m} \mathrm{~s}^{-1}$ & Estimated intrinsic RV jitter of the star. \\
\hline BASELINE & float32 & days & Maximum baseline of RV data included in fit \\
\hline SIGMA_V & float64 & $\mathrm{m} \mathrm{s}^{-1}$ & Median of RV errors, $\sigma_{v}$ \\
\hline SIG_RVVAR & float64 & $\ldots$ & Significance of the RV variations (see Section 3.1.2) \\
\hline $\mathrm{JD}$ & float64[50] & JD & Julian Date of observations included in fit \\
\hline $\mathrm{RV}$ & float $64[50]$ & $\mathrm{m} \mathrm{s}^{-1}$ & Radial velocities of observations included in fit \\
\hline RV_ERR & float64[50] & $\mathrm{m} \mathrm{s}^{-1}$ & Error in radial velocities of observations included in fit \\
\hline MODEL & float64[50] & $\mathrm{m} \mathrm{s}^{-1}$ & RVs of best-fit orbital model. \\
\hline RESID & float $64[50]$ & $\mathrm{m} \mathrm{s}^{-1}$ & Residuals of best-fit orbital model. \\
\hline PERIOD & float64 & days & Best-fit orbital period, $P$, of the system \\
\hline PERIOD_ERR & float64 & days & Uncertainty in $P$ \\
\hline SEMIAMP & float64 & $\mathrm{m} \mathrm{s}^{-1}$ & Best-fit RV semiamplitude, $K$, of the system \\
\hline SEMIAMP_ERR & float64 & $\mathrm{m} \mathrm{s}^{-1}$ & Uncertainty in $K$ \\
\hline ECC & float64 & $\ldots$ & Best-fit eccentricity, $e$, of the system \\
\hline ECC_ERR & float64 & $\cdots$ & Uncertainty in $e$ \\
\hline OMEGA & float64 & degrees & Argument of periastron, $\omega$ \\
\hline T0 & float64 & JD & Epoch of Transit \\
\hline TPERI & float64 & $\mathrm{JD}$ & Epoch of periastron \\
\hline V0 & float64 & $\mathrm{m} \mathrm{s}^{-1}$ & Intercept of the global trend applied to the RVs \\
\hline SLOPE & float64 & $\mathrm{m} \mathrm{s}^{-1}$ day $^{-1}$ & Slope of the global trend applied to the RVs \\
\hline
\end{tabular}


Table 4

(Continued)

\begin{tabular}{lccc}
\hline \hline Field Name & Data Type & Units & Description \\
\hline NITER & int16 & $\ldots$ & Number of iterations used to converge on a period (see Section 3.3.2) \\
CHI2 & float64 & $\ldots$ & $\chi^{2}$ (not reduced) of the fit \\
DOF & float64 & $\ldots$ & Degrees of freedom of the fit \\
FIT_RMS & float64 & $\mathrm{m} \mathrm{s}^{-1}$ & Root-Mean-Square of the residuals of the fit \\
PUI & float64 & $\ldots$ & Phase Uniformity Index (see Section 3.3.4) \\
VUI & float64 & $\ldots$ & Velocity Uniformity Index (see Section 3.3.4) \\
MASSFN & float64 & $M_{\odot}$ & Mass function of the system \\
MSINI & float64 & $M_{\odot}$ & Estimated $m$ sin $i$ of the companion \\
SEMIMAJ & float64 & AU & Estimated orbital semimajor axis of the companion \\
\hline
\end{tabular}

Notes.

${ }^{\text {a }}$ See http://www.sdss.org/dr12/algorithms/bitmasks/ for APOGEE bitmask definitions.

${ }^{\mathrm{b}}$ 0_calibrated ASPCAP, 1-uncalibrated ASPCAP, 2-RV mini-grid (see Section 3.2).

${ }^{\mathrm{c}}$ 0_-Torres et al. (2010) relation, 1-APOKASC, 2-RC, 3-spectrophotometric, 4-TRILEGAL, 5-young cluster distance (see Section 3.2).

(This table is available in its entirety in FITS format.)

1. APOGEE targeting information, 2MASS photometry, proper motions, and reduction flags.

2. Adopted APOGEE stellar parameters $\left(T_{\text {eff }}, \log g,[\mathrm{Fe} /\right.$ $\mathrm{H}]$ ), and estimates of each primary star's mass, radius, and distance, with flags indicating the source/quality of the stellar parameters and mass/radius/distance estimates.

3. Heliocentric RV measurements for each star derived using best-fit ASPCAP synthetic spectra as RV templates.

4. The best-fit orbital and physical parameters of each system's candidate companion.

These data are compiled into a FITS table, whose content is described in Table 4. The catalog is also available as a Filtergraph portal here: https://filtergraph.com/apOrbitPub. The Filtergraph portal also contains links to webpages containing plots of the RV curves for these systems. Additional data for each star, including spectra and additional photometry, are available publicly via SDSS DR12 (Alam et al. 2015). See http://www.sdss.org/dr12/ for instructions on the access and use of APOGEE DR12 data.

\section{B.1. Caveats}

Here we present some caveats regarding the quality of the data in this catalog:

1. All caveats that apply to all APOGEE data (Holtzman et al. 2015) also apply to this catalog.

2. Many stars with the longest baselines were observed during APOGEE commissioning, during which the instrument did not employ dithering. However, these stars were reobserved at the end of the survey with the standard instrument configuration, and RVs derived from commissioning data have been shown to be of similar quality to main-survey RVs.

3. The stellar parameters derived by ASPCAP for dwarf stars are uncalibrated, but good enough to establish estimates of the star's primary mass, and sufficiently accurate to distinguish between dwarfs and giants.

4. The RV errors output by the APOGEE reduction pipeline may be slightly underestimated. We refer the reader to Section 10.3 of Nidever et al. (2015) where RV uncertainties are discussed more fully.
5. The distances presented here are from a preliminary catalog, and will likely undergo future refinement.

6. The most common source of errors in the orbital parameters is the fitter choosing the wrong harmonic for the period. Therefore, the periods presented here may be an integer number ( 2 or 3$)$ or an integer fraction $(1 / 2$ or $1 / 3$ ) times the true period for the system. The fitter also had a tendency to inflate the eccentricities of the simulated systems, so the eccentricities, and thus the values of $K$ and $m \sin i$ presented here are likely to be slightly larger than their true values.

7. The values for argument and time of periastron $\left(T_{P}\right.$ and $\omega)$ become unconstrained at low eccentricities, and are poorly reproduced by this catalog. We release them so that our model curves can be reproduced, but should be taken with a grain of salt.

Finally we stress that the systems presented here are candidates, and that the orbital parameters presented here may only be characteristic of the true values of the system. In particular, the low-mass and low-visit candidates are the most in need of additional observation.

\section{REFERENCES}

Abt, H. A. 2008, AJ, 135, 722

Adamów, M., Niedzielski, A., Villaver, E., Nowak, G., \& Wolszczan, A. 2012, ApJL, 754, L15

Adibekyan, V. Z., Sousa, S. G., Santos, N. C., et al. 2012, A\&A, 545, A32

Alam, S., Albareti, F. D., Prieto, C. A., et al. 2015, ApJS, 219, 12

Beaulieu, J.-P., Bennett, D. P., Fouqué, P., et al. 2006, Natur, 439, 437

Bodaghee, A., Santos, N. C., Israelian, G., \& Mayor, M. 2003, A\&A, 404, 715

Bordé, P., Rouan, D., \& Léger, A. 2003, A\&A, 405, 1137

Borucki, W. J., Koch, D., Basri, G., et al. 2010, Sci, 327, 977

Bovy, J., Nidever, D. L., Rix, H.-W., et al. 2014, ApJ, 790, 127

Brucalassi, A., Pasquini, L., Saglia, R., et al. 2014, A\&A, 561, L9

Carlberg, J. K., Cunha, K., Smith, V. V., \& Majewski, S. R. 2012, ApJ, 757, 109

Carney, B. W., Latham, D. W., Stefanik, R. P., Laird, J. B., \& Morse, J. A. 2003, AJ, 125, 293

De Lee, N., Ge, J., Crepp, J. R., et al. 2013, AJ, 145, 155

Dotter, A., Chaboyer, B., Jevremović, D., et al. 2008, ApJS, 178, 89

Duchêne, G., \& Kraus, A. 2013, ARA\&A, 51, 269

Eggleton, P. P. 1983, ApJ, 268, 368

Eisenstein, D. J., Weinberg, D. H., Agol, E., et al. 2011, AJ, 142, 72

Fischer, D. A., \& Valenti, J. 2005, ApJ, 622, 1102

Fleming, S. W., Ge, J., Barnes, R., et al. 2012, AJ, 144, 72

Fleming, S. W., Mahadevan, S., Deshpande, R., et al. 2015, AJ, 149, 143 
Frink, S., Quirrenbach, A., Fischer, D., Röser, S., \& Schilbach, E. 2001, PASP, 113,173

García Pérez, A. E., Allende Prieto, C., Holtzman, J. A., et al. 2015, arXiv: 1510.07635

Ge, J., Mahadevan, S., Lee, B., et al. 2008, in ASP Conf. Ser. 398, Extreme Solar Systems, 449

Girardi, L., Groenewegen, M. A. T., Hatziminaoglou, E., \& da Costa, L. 2005, A\&A, 436, 895

Gizis, J. E., Kirkpatrick, J. D., Burgasser, A., et al. 2001, ApJL, 551, L163

Grether, D., \& Lineweaver, C. H. 2006, ApJ, 640, 1051

Guillot, T., Lin, D., Morel, P., Havel, M., \& Parmentier, V. 2014, EAS Publication Ser., 65, 327

Gunn, J. E., Siegmund, W. A., Mannery, E. J., et al. 2006, AJ, 131, 2332

Han, E., Wang, S. X., Wright, J. T., et al. 2014, PASP, 126, 827

Hayden, M. R., Bovy, J., Holtzman, J. A., et al. 2015, ApJ, 808, 132

Hekker, S., Snellen, I. A. G., Aerts, C., et al. 2008, A\&A, 480, 215

Herbig, G. H. 1998, ApJ, 497, 736

Hettinger, T., Badenes, C., Strader, J., Bickerton, S. J., \& Beers, T. C. 2015, ApJL, 806, L2

Holtzman, J. A., Shetrone, M., Johnson, J. A., et al. 2015, AJ, 150, 148

Jiang, P., Ge, J., Cargile, P., et al. 2013, AJ, 146, 65

Johnson, J. A., Fischer, D. A., Marcy, G. W., et al. 2007, ApJ, 665, 785

Jones, M. I., Jenkins, J. S., Bluhm, P., Rojo, P., \& Melo, C. H. F. 2014a, A\&A, 566, A113

Jones, M. I., Jenkins, J. S., Rojo, P., Melo, C. H. F., \& Bluhm, P. 2014b, A\&A, 573, A3

Kane, S. R., Henry, G. W., Dragomir, D., et al. 2011, ApJL, 735, L41

LaCourse, D. M., Jek, K. J., Jacobs, T. L., et al. 2015, MNRAS, 452, 3561

Lee, B. L., Ge, J., Fleming, S. W., et al. 2011, ApJ, 728, 32

Liebert, J., Bergeron, P., \& Holberg, J. B. 2005, ApJS, 156, 47

Lillo-Box, J., Barrado, D., Moya, A., et al. 2014, A\&A, 562, A109

Lovis, C., \& Mayor, M. 2007, A\&A, 472, 657

Ma, B., Ge, J., Barnes, R., et al. 2013, AJ, 145, 20

Mack, C. E., Ge, J., Deshpande, R., et al. 2013, AJ, 145, 139

Madore, B. F., \& Freedman, W. L. 2005, ApJ, 630, 1054

Majewski, S. R., Schiavon, R. P., Frinchaboy, P. M., et al. 2015, arXiv: 1509.05420

Marcy, G. W., \& Butler, R. P. 2000, PASP, 112, 137

Markwardt, C. B. 2009, adass XVIII, 411, 251

Massarotti, A., Latham, D. W., Stefanik, R. P., \& Fogel, J. 2008, AJ, 135, 209

Masset, F. S., \& Papaloizou, J. C. B. 2003, ApJ, 588, 494
Mathieu, R. D., Meibom, S., \& Dolan, C. J. 2004, ApJL, 602, L121

Matsuo, T., Shibai, H., Ootsubo, T., \& Tamura, M. 2007, ApJ, 662, 1282

Mayor, M., \& Queloz, D. 1995, Natur, 378, 355

Mészáros, S., Holtzman, J., García Pérez, A. E., et al. 2013, AJ, 146, 133

Mullally, F., Coughlin, J. L., Thompson, S. E., et al. 2015, ApJS, 217, 31

Nidever, D. L., Holtzman, J. A., Allende Prieto, C., et al. 2015, AJ, 150, 173

Palmer, D. M. 2009, ApJ, 695, 496

Penev, K., Jackson, B., Spada, F., \& Thom, N. 2012, ApJ, 751, 96

Pinsonneault, M. H., Elsworth, Y., Epstein, C., et al. 2014, ApJS, 215, 19

Raghavan, D., McAlister, H. A., Henry, T. J., et al. 2010, ApJS, 190, 1

Ramírez, I., \& Allende Prieto, C. 2011, ApJ, 743, 135

Reffert, S., Bergmann, C., Quirrenbach, A., Trifonov, T., \& Künstler, A. 2015, A\&A, 574, A116

Reffert, S., Quirrenbach, A., Mitchell, D. S., et al. 2006, ApJ, 652, 661

Ricker, G. R., Winn, J. N., Vanderspek, R., et al. 2014, Proc. SPIE, 9143 , 914320

Robinson, S. E., Laughlin, G., Bodenheimer, P., \& Fischer, D. 2006, ApJ, 643, 484

Rodrigues, T. S., Girardi, L., Miglio, A., et al. 2014, MNRAS, 445, 2758

Santiago, B. X., Brauer, D. E., Anders, F., et al. 2016, A\&A, 585, A42

Scargle, J. D. 1982, ApJ, 263, 835

Schultheis, M., Chen, B. Q., Jiang, B. W., et al. 2014, A\&A, 566, A120

Shetrone, M., Bizyaev, D., Lawler, J. E., et al. 2015, ApJS, 221, 24

Shvartzvald, Y., Maoz, D., Kaspi, S., et al. 2014, MNRAS, 439, 604

Silvotti, R., Charpinet, S., Green, E., et al. 2014, A\&A, 570, A130

Slawson, R. W., Prša, A., Welsh, W. F., et al. 2011, AJ, 142, 160

Tabachnik, S., \& Tremaine, S. 2002, MNRAS, 335, 151

Torres, G., Andersen, J., \& Giménez, A. 2010, A\&ARv, 18, 67

Tsang, D., Turner, N. J., \& Cumming, A. 2014, ApJ, 782, 113

Udalski, A. 2003, AcA, 53, 291

Villaver, E., \& Livio, M. 2009, ApJL, 705, L81

Villaver, E., Livio, M., Mustill, A. J., \& Siess, L. 2014, ApJ, 794, 3

Wisniewski, J. P., Ge, J., Crepp, J. R., et al. 2012, AJ, 143, 107

Wittenmyer, R. A., Endl, M., Wang, L., et al. 2011, ApJ, 743, 184

Wright, J. T., Roy, A., Mahadevan, S., et al. 2013, ApJ, 770, 119

Zamora, O., García-Hernández, D. A., Prieto, C. A., et al. 2015, AJ, 149, 181

Zasowski, G., Johnson, J. A., Frinchaboy, P. M., et al. 2013, AJ, 146, 81

Zieliski, P., Niedzielski, A., Wolszczan, A., Adamów, M., \& Nowak, G. 2012, A\&A, 547, A91 